\title{
Similarity in the far field of a turbulent round jet
}

\author{
P. Burattini ${ }^{\mathrm{a})}$ and R. A. Antonia \\ Discipline of Mechanical Engineering, University of Newcastle, Newcastle, N.S.W. 2308, Australia \\ L. Danaila \\ CORIA, Avenue de l'Université, BP12, 76801 Saint Etienne du Rouvray, France
}

(Received 2 May 2004; accepted 5 October 2004; published online 20 December 2004)

\begin{abstract}
In this paper, we test the idea of equilibrium similarity, for which all scales evolve in a similar way in a turbulent round jet, for a prescribed set of initial conditions. Similarity requirements of the mean momentum and turbulent energy equations are reviewed briefly but the main focus is on the velocity structure function equation, which represents an energy budget at any particular scale. For similarity of the structure function equation along the jet axis, it is found that the Taylor microscale $\lambda$ is the relevant characteristic length scale. Energy structure functions and spectra, measured at a number of locations along the axis of the jet, support this finding reasonably well, i.e., they collapse over a significant range of scales when normalized by $\lambda$ and the mean turbulent energy $\left\langle q^{2}\right\rangle$. Since the Taylor microscale Reynolds number $R_{\lambda}$ is approximately constant $(\simeq 450)$ along the jet axis, the structure functions and spectra also collapse approximately when the normalization uses either the Kolmogorov or integral length scales. Over the dissipative range, the best collapse occurs when Kolmogorov variables are used. The use of $\left\langle q^{2}\right\rangle$ and the integral length scale $L$ provides the best collapse at large separations. A measure of the quality of collapse is given. (C) 2005 American Institute of Physics. [DOI: 10.1063/1.1833414]
\end{abstract}

\section{INTRODUCTION}

The concept of self-preservation, which in essence assumes that the flow is governed by single length and velocity scales, has been of undeniable value for describing the spatial evolution of laminar and turbulent flows. From a mathematical viewpoint, reducing the partial differential equations, which govern the fluid motion, to ordinary differential equations represents a considerable advantage. The Blasius solution for a laminar boundary layer over a flat plate is a well known illustration of this. In the case of turbulent flows, self-preservation has met with only mixed success, partly because of the wide range of scales that are present in turbulent flows and also because the notion of forgetfulness, or disentanglement from initial conditions, which is implicit in the notion of self-preservation, is an oversimplification. There is now a sufficient amount of experimental evidence to suggest (see, e.g., George ${ }^{1}$ ) that initial conditions can influence the type of self-preservation that can be achieved in plane wakes (e.g., Wygnanski, Champagne, and Marasli ${ }^{2}$ and Antonia, Zhou, and Romano ${ }^{3}$ ) and also round jets. In the latter flow, different values - found in the literature-for the velocity decay rate in the far field and for the virtual origin point in this direction. George advanced the plausible suggestion that this influence reflects the link between selfpreservation and coherent structures.

At a more fundamental level, George argued that the previous difficulties, encountered with self-preservation, relate mainly to the restrictive manner in which the selfpreserving analysis was carried out. He showed that a more

\footnotetext{
a)Telephone: +6124921 8879. Fax: +61 24921 6946. Electronic mail: paolo.burattini@newcastle.edu.au
}

general analysis leads to the possibility that a particular type of flow can have a number of self-preserving states, each uniquely determined by its initial conditions. The new element in George's approach was to determine the relevant similarity scales from the analysis, instead of prescribing them arbitrarily from the outset.

George $^{1}$ applied the new analysis to both the plane wake and the axisymmetric jet by investigating the similarity requirements of the mean momentum and turbulent energy equations. The analysis was also applied to decaying homogeneous isotropic turbulence ${ }^{4}$ and a homogeneous uniform shear flow. ${ }^{5}$ The latter papers investigated the equilibrium similarity of the spectral energy equation; as for the momentum and mean turbulent energy equations, all terms in the spectral energy equation are required to evolve in exactly the same manner. In each case, the relevant length scale was found to be the Taylor microscale $\lambda$ with $\left\langle q^{2}\right\rangle^{1 / 2}$ (here taken as $\left\langle q^{2}\right\rangle \equiv u^{\prime 2}+2 v^{\prime 2}$, where $u^{\prime} \equiv\left\langle u^{2}\right\rangle^{1 / 2}$ and $v^{\prime} \equiv\left\langle v^{2}\right\rangle^{1 / 2}$ are the rms values of the streamwise and radial velocity fluctuations; the angular brackets denote averaging with respect to time and the prime represents the rms) as the characteristic velocity scale. Satisfactory support for this scaling was provided by measured spectra and velocity structure functions in grid turbulence ${ }^{6}$ and measured spectra in a constant mean shear flow (cf. George and Gibson ${ }^{5}$ and references therein). Since this similarity solution should apply to all scales of motion, the nonconstancy of $R_{\lambda} \equiv \lambda u^{\prime} / \nu$, where $\nu$ is the air viscosity and $\lambda \equiv u^{\prime} /(\partial u / \partial x)^{\prime}$ (for grid turbulence $R_{\lambda}$ decays with $x$; for a uniform shear flow, $R_{\lambda}$ increases exponentially with $x$ ), is not consistent with Kolmogorov's concept of local similarity (except possibly at infinite Reynolds number), i.e., the idea that the appropriate normalizing parameters for the 
small dissipative scales are the Kolmogorov length scale $\eta$ $\equiv\left(\nu^{3 / 4} /\langle\epsilon\rangle^{1 / 4}\right)$ and velocity scale $u_{K} \equiv\left(\nu^{1 / 4}\langle\epsilon\rangle^{1 / 4}\right)$, where $\langle\epsilon\rangle$ is the mean energy dissipation rate. A recent study (Antonia and Orlandi $^{7}$ ), based on direct numerical simulation (DNS) box turbulence data, indicated that the high wave number part of the velocity spectrum collapsed better when normalized by $\eta$ and $u_{K}$ than with $\lambda$ and $\left\langle q^{2}\right\rangle^{1 / 2}$. However, the latter pair of scales provides a good collapse for the remainder of the spectrum and is especially appropriate for calculating the nonlinear transfer of energy from large to small scales.

There are a few self-preserving flows (e.g., Tennekes and Lumley ${ }^{8}$ provide a detailed tabulation of free shear flows which are self-preserving) for which the Reynolds number should remain constant with $x$. For the plane wake, the mean velocity defect decays as $x^{-1 / 2}$ whereas the mean velocity half-width grows as $x^{1 / 2}$, so that the Reynolds number, defined on the basis of these quantities, is constant. For a round jet, discharging into still ambient surroundings, the mean velocity on the axis decays as $x^{-1}$, while the half-radius grows linearly with $x$. Another such flow is the boundary layer between converging plane walls ${ }^{9}$ for which the free stream velocity decays as $x^{-1}$ and the boundary layer thickness grows as $x$.

To our knowledge, there has been no previous attempt to test the similarity of the spectra or velocity structure functions in the above flows, even though the self-preserving forms of the mean velocity and Reynolds stress distributions have been well documented for the plane wake and round jet. Mathieu and Scott ${ }^{10}$ noted, in the specific context of the circular jet, that the smallest scales should form part of the similarity solution, given that the integral length scale should be proportional to $\eta$, at least for sufficiently large Reynolds numbers. Here, we focus on the circular jet, partly because relatively large values of $R_{\lambda}$ can be achieved for moderate values of the jet exit diameter and velocity, but also because the absence of lateral boundaries should minimize any interference on the largest scales of the flow.

Similarity of mean momentum and turbulent energy in the circular jet is reviewed only briefly in Sec. II, since it can be found in many textbooks. ${ }^{8,10,11}$ The equilibrium similarity of the structure functions is investigated in more detail in Sec. III. After providing relevant experimental details (Sec. IV) and a basic flow description (Sec. V), experimental support for the analysis is presented in Sec. VI. We consider measurements of both energy structure functions and onedimensional (1D) spectra corresponding to the turbulent energy at several locations along the axis of the jet. The investigation is restricted to the region along the axis where the influence of the terms involving the pressure-neglected in the mathematical analysis of Sec. III-is deemed negligible. To our knowledge, no measurements of the pressure related terms have been published. The DNS results of Boersma et $a l .{ }^{12}$ for the round jet do not clarify this point. Recently, DNS data were reported for a plane jet: ${ }^{13}$ The pressure diffusion terms were found to be negligible on the jet axis at the end of the computational domain (about 12 jet widths).

\section{SIMILARITY OF MEAN MOMENTUM AND TURBULENT ENERGY EQUATIONS}

Self-similarity solutions in the round jet can be sought, in a straightforward manner, through dimensional arguments. ${ }^{11,14}$ Alternatively (see, for instance, Ref. 10), one can assume a functional dependence for the mean streamwise velocity of the following type:

$$
U=U_{0}(x) \hat{f}(\zeta)
$$

where $U_{0}(x)$ is the axial streamwise velocity, $\hat{f}(\zeta)$ is the similarity profile for $U$, and $\zeta=\rho /\left(x-x_{0}\right)$ is the similarity variable ( $\rho$ is the radial coordinate). In this approach, we are assuming, from the outset, that there is just one characteristic length, viz. the distance $x$ from the virtual origin of $U_{0}, x_{0}$. From constancy of the momentum flux, viz.

$$
\int_{0}^{\infty} 2 \pi U^{2} \rho \mathrm{d} \rho=\text { const }
$$

it follows that

$$
U_{0}(x) \sim\left(x-x_{0}\right)^{-1},
$$

since $\rho \mathrm{d} \rho=\left(x-x_{0}\right)^{2} \zeta \mathrm{d} \zeta$. From the boundary-layer approximation to the first order, the momentum equations for the jet are

$$
\begin{aligned}
& \frac{1}{\rho} \frac{\partial(\rho V)}{\partial \rho}+\frac{\partial U}{\partial x}=0, \\
& U \frac{\partial U}{\partial x}+V \frac{\partial U}{\partial \rho}=-\frac{1}{\rho} \frac{\partial(\rho\langle u v\rangle)}{\partial \rho},
\end{aligned}
$$

from which the following functional forms for the radial velocity and the Reynolds shear stress:

$$
\begin{aligned}
& V \sim\left(x-x_{0}\right)^{-1} \hat{g}(\zeta), \\
& -\langle u v\rangle \sim\left(x-x_{0}\right)^{-2} \hat{h}(\zeta),
\end{aligned}
$$

can be obtained, where $\hat{g}$ and $\hat{h}$ are the similarity profiles for the radial mean velocity and Reynolds shear stress.

Conversely, if the characteristic length scale is not assumed to be linearly proportional to $x$ but is supposed instead to be a general function of $x$, say $\delta_{u}(x),{ }^{1,10}$ so that the general similarity variable is now $\zeta=\rho / \delta_{u}(x)$, the momentum conservation yields $U=\left[\delta_{u}(x)\right]^{-1} \hat{f}(\zeta)$, that is to say

$$
U_{0}(x) \sim\left[\delta_{u}(x)\right]^{-1} .
$$

From the boundary layer equations and the assumption that the mean and turbulent velocities have the same dependence on $x$, viz.

$$
-\langle u v\rangle=\delta_{u v}(x)^{-2} \hat{h}(\zeta),
$$

with $\delta_{u v}(x) \sim \delta_{u}(x)$, it follows that

$$
\frac{\mathrm{d} \delta_{u}}{\mathrm{~d} x}=\text { constant, }
$$

so that $\delta_{u}$ (and $\delta_{u v}$ ) is linear with $x$

The assumption $\delta_{u v}(x) \sim \delta_{u}(x)$ can be avoided altogether by considering the transport equations for the individual 
Reynolds stress components along the axis of the jet (e.g., Appendix C in Ref. 15). If a similarity form is assumed for each of the terms in these equations, it can be rigorously demonstrated that the length scale $\delta_{u}$ has to increase linearly along the streamwise direction, for the equations to admit to similarity solutions ${ }^{16}$ (see also Ref. 17 for the plane jet). This development hinges also on the fact that the sum of the pressure-strain terms in the equations for the Reynolds normal stresses is zero for an incompressible flow.

\section{SIMILARITY OF ENERGY STRUCTURE FUNCTION EQUATION}

The far field of the circular jet evolves spatially, in terms of mean and fluctuating quantities, according to the criteria set out in the previous section. As a further step, it is of interest to investigate the similarity of all possible scales contributing to the energy, not just the largest ones or those associated with the dissipation of energy. This approach, referred to as equilibrium similarity by George, ${ }^{4}$ contrasts with that which addresses only a localized range of scales, such as the local similarity concept of Kolmogorov. ${ }^{18}$ The evolution of spectra or second-order structure functions of the velocity is typically used for this purpose, since both sift out the distribution of the energy among the different scales. In this section, we investigate the consequences of applying similarity to the velocity structure function equation. Experimental corroboration will be presented later (Secs. VI A and VI B), for both structure functions and spectra.

We consider both these quantities since, even though they are related-the formal relationship involves a cosine transform, see for instance, Ref. 19-the treatment, in physical space, is more amenable to experimental verification. We thus avoid having to deal with three-dimensional (3D) energy spectra and energy transfer functions. The evolution equation for the structure functions on the axis of a circular jet was recently derived from the Navier-Stokes equations by Danaila et $a .^{20}$ It was assumed that local isotropy was satisfied by the turbulent advection, molecular diffusion, and pressure diffusion terms and the effect of the large scale inhomogeneity was treated in a quasi-isotropic manner. In a recent paper, ${ }^{6}$ a similar approach, starting with the structure function equation for grid turbulence in Ref. 21, was applied to experimental data. For that flow, which is arguably the simplest one available since there is no mean shear and hence no turbulent energy production, the experimental verification of the scale-by-scale energy budget is particularly satisfactory. There was also quite adequate support for $\lambda$ and $\left\langle q^{2}\right\rangle^{1 / 2}$ as the appropriate similarity parameters over a significant range of length scales.

Along the jet axis, the transport equation for the turbulent energy is given by ${ }^{20}$

$$
\begin{gathered}
-\left\langle\delta u(\delta q)^{2}\right\rangle+2 \nu \frac{\mathrm{d}}{\mathrm{d} r}\left\langle(\delta q)^{2}\right\rangle-\frac{U_{0}}{r^{2}} \int_{0}^{r} s^{2} \frac{\partial}{\partial x}\left\langle(\delta q)^{2}\right\rangle \mathrm{d} s \\
-2 \frac{\partial U_{0}}{\partial x} \frac{1}{r^{2}} \int_{0}^{r} s^{2}\left(\left\langle(\delta u)^{2}\right\rangle-\left\langle(\delta v)^{2}\right\rangle\right) \mathrm{d} s=\frac{4}{3}\langle\epsilon\rangle_{\mathrm{hom}} r,
\end{gathered}
$$

where $s$ is a dummy variable, $r$ is the spatial separation over which the increments are evaluated. The first term in Eq. (7) is the generalized third-order structure function, while the second represents the viscous effect. The third and fourth terms quantify the role of the streamwise inhomogeneity and of the turbulent energy production, respectively. Finally, the term on the right side of Eq. (7) is proportional to the energy dissipation rate and balances the sum of the other terms. Here, we use a homogeneous form of the mean energy dissipation rate $\left(\langle\epsilon\rangle_{\text {hom }} \equiv 3 \nu\left[\left\langle(\partial u / \partial x)^{2}\right\rangle+2\left\langle(\partial v / \partial x)^{2}\right\rangle\right]\right.$, which is more appropriate than $\langle\epsilon\rangle_{\text {iso }} \equiv 15 \nu\left\langle(\partial u / \partial x)^{2}\right\rangle$, since $2\left\langle(\partial u / \partial x)^{2}\right\rangle$ is substantially larger than $\left\langle(\partial v / \partial x)^{2}\right\rangle$, along the jet axis, as can be seen in Table III (see also Sec. V for further discussion).

In order to examine the conditions under which Eq. (7) satisfies similarity, we need to assume functional forms for the terms in this relation. Following Antonia et al., ${ }^{6}$ we assume

$$
\begin{aligned}
& \left\langle(\delta q)^{2}\right\rangle=Q(x) f(\xi), \\
& \left\langle(\delta u)^{2}\right\rangle=M(x) e(\xi), \\
& \left\langle(\delta v)^{2}\right\rangle=R(x) h(\xi), \\
& -\left\langle\delta u(\delta q)^{2}\right\rangle=T(x) g(\xi),
\end{aligned}
$$

where $\xi=r / \ell$ and $\ell$ is a characteristic length scale, yet to be determined. A possible dependence on the initial conditions, as explained in Ref. 4, is also plausible, but is not considered here explicitly. $Q(x),{ }^{49} M(x), R(x)$, and $T(x)$ are scales that characterize the second-order structure functions of $q, u, v$, and the third-order structure function $\left\langle\delta u(\delta q)^{2}\right\rangle$, respectively. The lower-case functions represent the shape of the structure functions. The separation between functions of $x$ and $\xi$ allows the determination of solutions to the transport equation, for which a relative balance among all of the terms is maintained as the flow progresses downstream. After substituting Eqs. (8)-(11) into Eq. (7), we obtain (after differentiating and rearranging and considering only the variations in $x$ and $r$ )

$$
\begin{gathered}
T(x) g(\xi)+2 \nu Q(x) \frac{1}{\ell} \frac{\mathrm{d} f(\xi)}{\mathrm{d} \xi}-\frac{U_{0}}{r^{2}} \frac{\mathrm{d} Q(x)}{\mathrm{d} x} \ell^{3} \Gamma_{1} \\
+\frac{U_{0} Q(x)}{r^{2}} \frac{\mathrm{d} \ell}{\mathrm{d} x} \ell^{2} \Gamma_{2}-2 \frac{\mathrm{d} U_{0}}{\mathrm{~d} x} \frac{M(x)}{r^{2}} \ell^{3} \Gamma_{3} \\
+2 \frac{\partial U_{0}}{\partial x} \frac{R(x)}{r^{2}} \ell^{3} \Gamma_{4}=\frac{4}{3}\langle\epsilon\rangle_{\mathrm{hom}} r,
\end{gathered}
$$

where

$$
\begin{aligned}
& \Gamma_{1}=\int_{0}^{r / \ell} \frac{s^{2}}{\ell^{2}} f(\xi) \mathrm{d}\left(\frac{s}{\ell}\right), \\
& \Gamma_{2}=\int_{0}^{r / \ell} \frac{s^{3}}{\ell^{3}} \frac{\mathrm{d} f(\xi)}{\mathrm{d} \xi} \mathrm{d}\left(\frac{s}{\ell}\right), \\
& \Gamma_{3}=\int_{0}^{r / \ell} \frac{s^{2}}{\ell^{2}} e(\xi) \mathrm{d}\left(\frac{s}{\ell}\right),
\end{aligned}
$$




$$
\Gamma_{4}=\int_{0}^{r / \ell} \frac{s^{2}}{\ell^{2}} h(\xi) \mathrm{d}\left(\frac{s}{\ell}\right)
$$

Note that the following relation:

$$
\frac{\partial \xi}{\partial x}=-r \ell^{-2} \frac{\mathrm{d} \ell}{\mathrm{d} x}
$$

has been used in deriving Eq. (12). After separating the terms which depend on $x$ from those which depend on $\xi$, and multiplying by $(\ell / \nu Q(x))$, Eq. (12) becomes

$$
\begin{gathered}
{\left[\frac{T(x) \ell}{\nu Q(x)}\right] g(\xi)+[2] \frac{\mathrm{d} f(\xi)}{\mathrm{d} \xi}-\left[\frac{U_{0} \ell^{2}}{\nu Q(x)} \frac{\mathrm{d} Q(x)}{\mathrm{d} x}\right] \frac{\Gamma_{1}}{\xi^{2}}} \\
+\left[\frac{U_{0}}{\nu} \frac{\mathrm{d} \ell}{\mathrm{d} x} \ell\right] \frac{\Gamma_{2}}{\xi^{2}}-\left[2 \frac{\mathrm{d} U_{0}}{\mathrm{~d} x} \frac{M(x)}{\nu Q(x)} \ell^{2}\right] \frac{\Gamma_{3}}{\xi^{2}} \\
+\left[2 \frac{\mathrm{d} U_{0}}{\mathrm{~d} x} \frac{R(x)}{\nu Q(x)} \ell^{2}\right] \frac{\Gamma_{4}}{\xi^{2}}=\left[\frac{4}{3} \frac{\langle\epsilon\rangle_{\mathrm{hom}} \ell^{2}}{\nu Q(x)}\right] \xi .
\end{gathered}
$$

For equilibrium similarity, all terms within the square brackets must evolve in the streamwise direction in the same manner. Since the second of these is constant, all the others must also be constant, viz.

$$
\begin{aligned}
& \frac{T \ell}{\nu Q}=\text { const, } \\
& \frac{U_{0} \ell^{2}}{Q} \frac{\mathrm{d} Q}{\mathrm{~d} x}=\text { const, } \\
& U_{0} \frac{\mathrm{d} \ell}{\mathrm{d} x} \ell=\mathrm{const}, \\
& \frac{\mathrm{d} U_{0}}{\mathrm{~d} x} \frac{M}{Q} \ell^{2}=\text { const, } \\
& \frac{\mathrm{d} U_{0}}{\mathrm{~d} x} \frac{R}{Q} \ell^{2}=\text { const, } \\
& \frac{\langle\epsilon\rangle_{\mathrm{hom}} \ell^{2}}{Q}=\text { const }
\end{aligned}
$$

(where the dependence on $x$ has been dropped for simplicity).

Equation (18) suggests that $\ell$ can be identified with $\lambda_{q}$ (hereafter, for simplicity, the exponent 2 in $q^{2}$ has been dropped when it appears as a subscript), which is defined as $\left(\left\langle q^{2}\right\rangle /\langle\epsilon\rangle_{\mathrm{hom}}\right)^{1 / 2}$, since $Q(x)$ represents the limiting value (or $\left.2\left\langle q^{2}\right\rangle\right)$ of $\left\langle(\delta q)^{2}\right\rangle$ for large separations. The relevant length scale which emerges from the above analysis is the Taylor microscale $\lambda_{q}$. As has already been found for decaying homogeneous isotropic turbulence ${ }^{4,6}$ and the homogeneous shear flow, ${ }^{5,20}$ the Taylor microscale appears to be the length scale relevant to the whole of the energy spectrum. This relevance apparently reflects the fact that $\lambda_{q}$ combines information about both large and small scale quantities, and negates the criticism ${ }^{8}$ that $\lambda_{q}$ has no direct physical meaning. This is, perhaps, not surprising given the rather complex role of $\langle\epsilon\rangle$, which represents the rate at which energy is dissipated over small scales, but may also be interpreted as the rate at which the energy is injected at large scales as well as the rate of transfer of the energy down the cascade, from large to small scales. Note that the scale-by-scale budget Eq. (7) reduces to the transport equation for $\left\langle q^{2}\right\rangle$ in the limit of $r \rightarrow \infty$. In the limit of $r \rightarrow 0$, it reduces to the transport equation for the enstrophy (or the mean energy dissipation rate $\langle\epsilon\rangle$ ).

\section{EXPERIMENTAL DETAILS}

The jet is generated by an open circuit wind tunnel equipped with a variable speed twelve-blade centrifugal blower. The tunnel comprises a diffuser, a settling chamber and a contraction with an area ratio of 85:1. A flexible connection is inserted between the blower and the rest of the tunnel in order to reduce motor-induced vibrations. Several screens and a honeycomb are fitted inside the settling chamber to reduce the turbulence level and to straighten the flow. The jet exits through a nozzle having a diameter $D=2 R$ $=55 \mathrm{~mm}$ in a large laboratory room whose temperature remained fairly constant $\left( \pm 0.5^{\circ} \mathrm{C}\right)$ throughout every single set of tests. The traversing system allows three degrees of freedom in the streamwise $(x)$, lateral $(y)$, and vertical $(z)$ directions with a resolution of $1,0.02$, and $0.01 \mathrm{~mm}$, respectively. Hereafter, the results will refer to this coordinate system and data for the streamwise and lateral (or radial, $\rho$ ) directions will be presented-in which the fluctuating velocity components are $u$ and $v$. The corresponding uppercase quantities, $U$ and $V$, are time-averaged values.

The velocity data were acquired with in-house hot wires and DISA anemometers (55M01 model). Both single and $\mathrm{X}$-wire probes were used, the latter with an angle between the wires of nearly $90^{\circ}$ and a lateral separation between the wires of approximately $0.8 \mathrm{~mm}$. The hot wires were etched from Pt-10\% Rh to a diameter of $d_{w}=2.5 \mu \mathrm{m}$ and the active length $l_{w}$ was chosen so as to have an aspect ratio $l_{w} / d_{w}$ of nearly 200 .

Velocity and angle calibrations were carried out in situ at the jet exit plane. For the speed calibration of the single wire, a third-order polynomial law was fitted to the anemometer output voltage as a function of the mean velocity. The $\mathrm{X}$-wire was calibrated at several values of speed and angle in the ranges of $\pm 40^{\circ}$ (in steps of $10^{\circ}$ ) and $0.9-17.2 \mathrm{~ms}^{-1}$, respectively. This set of values was used as a look-up-table (LUT), during data reduction, to estimate the velocity through spline interpolations. The LUT method was verified to perform better than an effective angle method, ${ }^{22}$ which assumed a constant sensibility coefficient for the lateral velocity. The percentage of drop-outs was always less than $1.2 \%$. The exit velocity of the flow was inferred from a Pitot tube connected to a Furness FCO12 micromanometer (full scale $=20 \mathrm{mmH}_{2} \mathrm{O}$, least count $=0.048 \mathrm{mmH}_{2} \mathrm{O}$ ). The single wire data were used as a check of the $\mathrm{X}$-wire response and differences in the mean values of the velocity were always below $2 \%$. The anemometer signals were acquired by means of a 16-bit AD board into a PC. Errors in the main turbulent quantities, given in Table I, were calculated by repeating the measurements 30 times at $x=60 \mathrm{D}$ and applying the error 
TABLE I. Uncertainties in several turbulence quantities (percentage values).

\begin{tabular}{ccccccc}
\hline \hline$U$ & $u^{\prime 2}$ & $v^{\prime 2}$ & $\left\langle(\partial u / \partial x)^{2}\right\rangle$ & $\left\langle(\partial v / \partial x)^{2}\right\rangle$ & $R_{\lambda}$ & $R_{\lambda q}$ \\
\hline 0.8 & 4.3 & 3.3 & 6.9 & 4.6 & 5.2 & 2.7 \\
\hline
\end{tabular}

estimation with 1:20 odds. ${ }^{23}$ Basic flow parameters are given in Table II, while some velocity statistics for several values of $x / D$ are reported in Table III.

\section{A. Correction for the spatial resolution of the $\mathrm{X}$-wire}

The X-wire has a finite spatial resolution dictated by both the length of the sensitive (etched) part of the wire and the distance between the two wires. A quite general procedure for correcting the spatial attenuation of this probe was given by Wyngaard. ${ }^{24}$ The treatment was developed in spectral space since it is easier to account for the integration effect on Fourier components of the velocity. To assess the attenuation function, the shape of the three-dimensional energy spectrum of the velocity needs to be assumed. Here, we have chosen the form used by Martinez et al. ${ }^{25}$ and the attenuation function was evaluated in accordance with the procedure of Zhu and Antonia, ${ }^{26}$ who extended Wyngaard's original analysis. ${ }^{24}$ This procedure takes into account the effects of the included angle between the wires, the length of the wires and the separation between the wires, and assumes the form of a correction function which is applied to the spectral content of the velocity and, consequently, the energy dissipation rate, for $u$ and $v$. The spectral correction was applied after the velocity data were re-sampled according to the modified Taylor's hypothesis, which is described in some detail below. Corrections for $u^{\prime}$ and $v^{\prime}$, due to spatial attenuation, were within $0.7 \%$ of the original values, while the values of $(\partial u / \partial x)^{\prime 2}$ were corrected upwards from $10 \%$ to $2 \%$ (between $x / D=30$ to $x / D=90$, respectively) and the values $(\partial v / \partial x)^{\prime 2}$ from $26 \%$ to $6 \%$, over the same range of $x / D$. The radial component is, thus, more affected by the spectral attenuation correction, for the X-probe configuration we used.
TABLE II. Some parameters of the jet (the values of the turbulence Reynolds numbers are averages over the range $35 \leqslant x / D \leqslant 90$ ).

\begin{tabular}{ccccc}
\hline \hline $\begin{array}{c}U_{j} \\
\left(\mathrm{~ms}^{-1}\right)\end{array}$ & $\begin{array}{c}R_{D} \\
-\end{array}$ & $\begin{array}{c}\nu \\
\mathrm{m}^{2} \mathrm{~s}^{-1}\end{array}$ & $\begin{array}{l}R_{\lambda} \\
-\end{array}$ & $\begin{array}{c}R_{\lambda q} \\
-\end{array}$ \\
\hline 35.1 & $1.3 \times 10^{5}$ & $1.5 \times 10^{-5}$ & 450 & 363 \\
\hline \hline
\end{tabular}

\section{B. Effect of modifying Taylor's hypothesis}

The present hot wire measurements have been done with a static hot wire, i.e., the time-varying velocity signals were acquired with probes at a fixed location. To obtain information in terms of $x$ instead of $t$, a time-space transformation is invoked. This is usually performed via Taylor's hypothesis, viz.

$$
u_{i}(x, t)=u_{i}(x-U \tau, t+\tau),
$$

when the delay $\tau$ is not too large (in this section the three velocity components are sometimes indicated with numbered subscripts). In this case, time and space derivatives are related by

$$
u_{i, t}=-u_{i, 1} U_{1},
$$

where repeated indices imply summation, a comma indicates differentiation and $i=(1,2,3)$. This approximationassuming that $U$ can be replaced by the mean streamwise velocity $U_{1}$-is acceptable for small turbulence intensities, $u_{i}^{\prime} / U$, since, in this case, the turbulence can be thought to be advected by a constant convection velocity. In the far field of a jet, the turbulence intensity is about $25 \%$ and such an approximation can lead to large errors, since the velocity fluctuations are high enough to render the concept of uniform translation questionable. Flying-hot-wires are sometimes used to alleviate this problem by introducing an additive transport velocity, thus fictitiously reducing the turbulence intensity. It is not practical to use flying-hot-wires because of the complications involved in displacing the probe at sufficiently high velocities and performing ensemble averages.

\begin{tabular}{|c|c|c|c|c|c|c|c|c|}
\hline $\begin{array}{l}x / D \\
-\end{array}$ & $\begin{array}{c}U_{0} \\
\left(\mathrm{~ms}^{-1}\right)\end{array}$ & $\begin{array}{c}u^{\prime} \\
\left(\mathrm{ms}^{-1}\right)\end{array}$ & $\begin{array}{c}v^{\prime} \\
\left(\mathrm{ms}^{-1}\right)\end{array}$ & $\underline{u^{\prime} / v^{\prime}}$ & $\begin{array}{c}\left\langle q^{2}\right\rangle \\
\left(\mathrm{m}^{2} \mathrm{~s}^{-2}\right)\end{array}$ & $\begin{array}{c}\left\langle(\partial u / \partial x)^{2}\right\rangle \\
\left(\mathrm{s}^{-2}\right) \times 10^{-5}\end{array}$ & $\begin{array}{c}\left\langle(\partial v / \partial x)^{2}\right\rangle \\
\left(\mathrm{s}^{-2}\right) \times 10^{-5}\end{array}$ & $\underline{I}$ \\
\hline 30 & 7.9 & 1.8 & 1.4 & 1.24 & 7.1 & 1.9 & 3.4 & 1.8 \\
\hline 35 & 6.7 & 1.5 & 1.2 & 1.21 & 5.4 & 1.2 & 2.3 & 1.9 \\
\hline 40 & 5.9 & 1.3 & 1.1 & 1.21 & 3.9 & 0.69 & 1.2 & 1.8 \\
\hline 45 & 5.2 & 1.1 & 0.94 & 1.21 & 3.1 & 0.43 & 0.7 & 1.7 \\
\hline 50 & 4.6 & 1.0 & 0.82 & 1.23 & 2.4 & 0.26 & 0.44 & 1.7 \\
\hline 55 & 4.2 & 0.92 & 0.77 & 1.20 & 2.0 & 0.20 & 0.30 & 1.6 \\
\hline 60 & 3.8 & 0.86 & 0.71 & 1.22 & 1.7 & 0.13 & 0.21 & 1.7 \\
\hline 65 & 3.5 & 0.78 & 0.65 & 1.20 & 1.4 & 0.094 & 0.15 & 1.6 \\
\hline 70 & 3.2 & 0.74 & 0.60 & 1.24 & 1.3 & 0.072 & 0.12 & 1.7 \\
\hline 75 & 3.0 & 0.70 & 0.57 & 1.22 & 1.2 & 0.056 & 0.10 & 1.8 \\
\hline 80 & 2.8 & 0.67 & 0.51 & 1.30 & 0.97 & 0.039 & 0.066 & 1.7 \\
\hline 85 & 2.6 & 0.61 & 0.49 & 1.26 & 0.85 & 0.035 & 0.057 & 1.6 \\
\hline 90 & 2.4 & 0.58 & 0.46 & 1.27 & 0.77 & 0.026 & 0.042 & 1.6 \\
\hline
\end{tabular}

TABLE III. Results for the velocity scales on the jet axis. 
Velocity spectra in the far field of the jet requires long and continuous velocity signals at a fixed location and the flyinghot-wire technique is in conflict with these requirements. In the present work, a modified version of Taylor's hypothesis, Eq. (19), is used. The time derivative of $u_{i}$ is approximated by

$$
u_{i, t}=-u_{i, 1} U_{1}-u_{j} u_{i, j}
$$

where $j=(1,2,3)$. With the further assumption that the spatial derivatives of the velocity fluctuations in directions 2 and 3 are negligible, we obtain

$$
\begin{aligned}
& u_{1, t}=-u_{1,1} U_{1}-u_{1} u_{1,1}=-u_{1,1}\left(U_{1}+u_{1}\right), \\
& u_{2, t}=-u_{2,1} U_{1}-u_{1} u_{2,1}=-u_{2,1}\left(U_{1}+u_{1}\right), \\
& u_{3, t}=-u_{3,1} U_{1}-u_{1} u_{3,1}=-u_{3,1}\left(U_{1}+u_{1}\right) .
\end{aligned}
$$

This approach, which is hereafter referred to as modified Taylor's hypothesis or MTH, differs somewhat from that of Lumley $^{27}$ and Heskestad, ${ }^{28}$ who used a large scale convection velocity. We have found that the use of a large scale convection velocity resolves in only slight changes in the high wave number part of the $u$ and $v$ spectra, compared to the use of the instantaneous convection velocity. For example, $\left\langle(\partial u / \partial x)^{2}\right\rangle$ and $\left\langle(\partial v / \partial x)^{2}\right\rangle$ are approximately $2 \%$ and $1.4 \%$ larger, when the large scale convection velocity is used.

The time-space transformation of velocity derivatives amounts to resampling (unevenly) the original (evenly spaced by $\Delta x=U_{1} \Delta t$, where $\Delta t$ is the inverse of the sampling frequency) velocity signals at the new locations given by

$$
x_{k}^{\prime}=\sum_{k=1}^{N}\left(U_{1}\left(t_{k}\right)+u_{1}\left(t_{k}\right)\right) \Delta t
$$

where $N$ is the number of samples and $t_{k}=k \Delta t$. Here, the "new" velocity values at $x_{k}^{\prime}$ were obtained with a locally linear interpolation. This resampling procedure produces signals with similar characteristics to laser Doppler velocimetry (LDV) signals, where the particle arrival times are not evenly spread but approximately follow a (Poisson) probability distribution. The effect on the statistics of the velocity fluctuations can be significant, as illustrated in Fig. 1, which shows $k_{1}^{2} \phi_{u}\left(k_{1}\right)$, the $1 \mathrm{D}$ energy dissipation rate spectrum based on $u$ [similar results, not shown here, hold for $k_{1}^{2} \phi_{v}\left(k_{1}\right)$ ]. The distribution calculated after correcting for the spatial attenuation is also reproduced. The major effects are concentrated at small scales, where Taylor's hypothesis considerably overestimates the distribution (see also the inset showing the energy spectra). The spatial attenuation correction acts in the opposite direction, increasing the measured values at high wave numbers, but is of minor importance, compared to the effect of MTH. The measured isotropy of the small scales (ratio of the energy dissipation rates estimated from the two velocity components) is improved by the application of MTH and the spectral attenuation correction.

The structure functions presented in this paper have been calculated from velocity signals corrected using the MTH. However, no spatial attenuation correction was applied to the

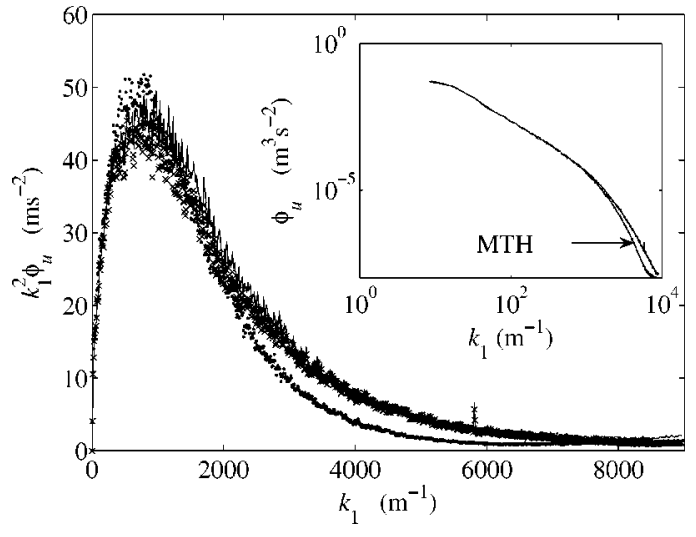

FIG. 1. Spectrum of the energy dissipation rate of $u: \times$, applying Taylor's hypothesis; - , corrected for spatial attenuation after applying Taylor's hypothesis; ', corrected for spatial attenuation after applying modified Taylor's hypothesis (MTH). The inset shows the spectrum of $u$ according to Taylor's hypothesis and MTH.

structure functions since a procedure equivalent to that previously outlined for the spectra is not available in physical space.

The algorithm for applying the MTH is similar to that used by Kahalerras et al. ${ }^{29}$ for assessing the effect the correction has on the velocity spectrum and pdf. Our results corroborate their conclusion regarding the major effect, especially at small scales, of this correction. The application of the correction for spectral attenuation and particularly the MTH was verified here to be important for obtaining reliable information on the streamwise development of turbulence quantities, in particular those based on $\langle\epsilon\rangle$, such as $\lambda$ and $R_{\lambda}$.

\section{BASIC FLOW PARAMETERS}

The jet issues from a round nozzle in a nominally laminar state with a turbulence intensity of nearly $1.5 \%$, mainly due to the low-frequency unsteadiness of the fan, and a tophat velocity profile. The exit velocity is $U_{j} \simeq 35 \mathrm{~ms}^{-1}$ which yields a Reynolds number of $R e_{D}=1.3 \times 10^{5}$. More details on the state of the boundary layer can be found in Ref. 30. In the far field, $x / D \geqslant 30$, the mean streamwise velocity on the axis, $U_{0}$, decays as shown in Fig. 2 (where data from other sources, ${ }^{15,31}$ are also included). The decay of $U_{j} / U_{0}$ is almost linear and the least-squares fit to the data (the residual, viz. the sum of the deviations from this fit, is 0.14), based on the relation $U_{j} / U_{0}=C^{-1}\left(x-x_{0}\right) / D$, gives a virtual origin $x_{0}$ of $4.4 D$ and a value of 6.0 for $C$. These values are similar to those in Ref. $15\left(C=5.8, x_{0} / D=4.4\right.$ from LDV measurements and $C=5.9, x_{0} / D=2.7$ from static hot wire measurements) for a jet at $R e_{D} \simeq 10^{5}$ issuing with a top-hat velocity profile in a nominally laminar state. The decay rate constant is also close to that $(C=6.1)$ measured with a flying hot wire in Ref. 32 with $R e_{D} \simeq 1.1 \times 10^{4}$. These values for $C$ seem to confirm the expectation that the mean velocity measurements are not too affected by the technique adopted. The linear decay rate of $U_{0}^{-1}$ along the axis is a consequence of the global similarity of the round jet, as discussed in Sec. II. Similarity of different order statistics is achieved gradually as the flow progresses downstream: while the collapse of 


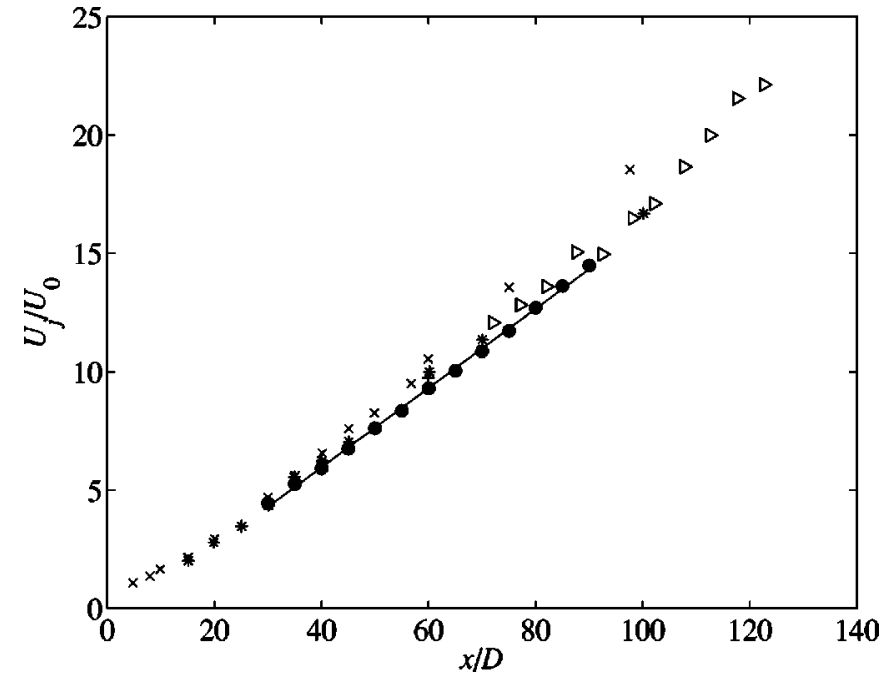

FIG. 2. Variation of the mean streamwise velocity on the jet axis: $\bullet$, Present data $R e_{D}=1.3 \times 10^{5}$; solid line: least squares fit to present data; $\times$, static hot wire (Ref. 31 ), $R e_{D} \simeq 10^{5}$; + , flying hot wire (Ref. 15$), R e_{D} \simeq 10^{5}$; *, static hot wire (Ref. 15), $R e_{D} \simeq 10^{5}$; $\triangleright$, LDA (Ref. 15), $R e_{D} \simeq 10^{5}$.

radial profiles of the streamwise velocity appears as soon as $x / D=8$ (Ref. 8 , p. 130), the linear decay of the mean velocity is expected to apply beyond $x / D \simeq 20{ }^{10}$ or $30 .{ }^{19}$ Turbulence quantities are reported to be still evolving at $x / D \simeq 40 .{ }^{8}$ Small differences, from one experimental setup to another, in the coefficients of the decay may be attributed to the effects of the initial conditions and $R e_{D}{ }^{1}$

Axial profiles of the streamwise turbulence intensity, $u^{\prime} / U_{0}$, and the ratios $u^{\prime} / v^{\prime}$ and $L_{u} / L_{v}$ are plotted in Fig. 3, where

$$
L_{\alpha}=\int_{0}^{r_{0}} \rho_{\alpha \alpha}(r) \mathrm{d} r
$$

is the integral length scale of $\alpha(\equiv\{u, v\})$. The upper limit of the integration $r_{0}$ corresponds to the location of the first zerocrossing of the correlation coefficient,

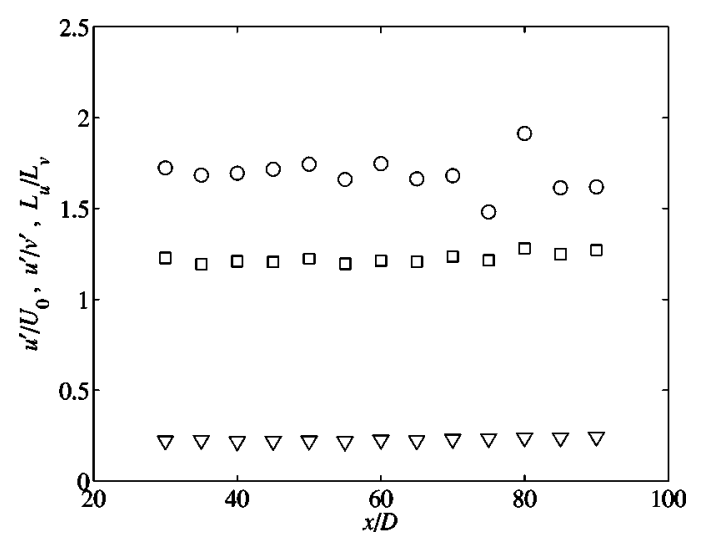

FIG. 3. Variation along jet axis of rms values of $u$ and $v$, and ratio of integral length scales associated with $u$ and $v: \nabla, u^{\prime} / U_{0} ; \square, u^{\prime} / v^{\prime} ; \bigcirc$, $L_{u} / L_{v}$.
TABLE IV. Length scales on the jet axis.

\begin{tabular}{ccccccccc}
\hline \hline$x / D$ & $\begin{array}{c}\eta \\
-\end{array}$ & $\begin{array}{c}\lambda \\
(\mathrm{mm})\end{array}$ & $\begin{array}{c}\lambda_{q} \\
(\mathrm{~mm})\end{array}$ & $\begin{array}{c}L_{u} \\
(\mathrm{~m})\end{array}$ & $\begin{array}{c}L_{v} \\
(\mathrm{~m})\end{array}$ & $\begin{array}{c}L_{q} \\
(\mathrm{~m})\end{array}$ & $\begin{array}{c}\lambda_{q} / \eta \\
-\end{array}$ & $\begin{array}{c}L_{q} / \lambda_{q} \\
-\end{array}$ \\
\hline 30 & 0.096 & 4.0 & 3.7 & 0.060 & 0.035 & 0.043 & 38 & 12 \\
35 & 0.11 & 4.3 & 3.9 & 0.071 & 0.042 & 0.051 & 37 & 13 \\
40 & 0.12 & 4.9 & 4.6 & 0.079 & 0.047 & 0.057 & 37 & 12 \\
45 & 0.14 & 5.5 & 5.2 & 0.092 & 0.053 & 0.065 & 37 & 13 \\
50 & 0.16 & 6.3 & 5.9 & 0.10 & 0.060 & 0.074 & 37 & 12 \\
55 & 0.18 & 6.6 & 6.4 & 0.11 & 0.067 & 0.080 & 37 & 12 \\
60 & 0.19 & 7.7 & 7.3 & 0.12 & 0.070 & 0.085 & 38 & 12 \\
65 & 0.21 & 8.0 & 7.8 & 0.12 & 0.071 & 0.086 & 37 & 11 \\
70 & 0.22 & 8.7 & 8.2 & 0.15 & 0.088 & 0.11 & 37 & 13 \\
75 & 0.23 & 9.3 & 8.6 & 0.15 & 0.098 & 0.11 & 37 & 13 \\
80 & 0.26 & 10.7 & 9.7 & 0.19 & 0.098 & 0.13 & 38 & 13 \\
85 & 0.27 & 10.4 & 9.8 & 0.17 & 0.10 & 0.12 & 37 & 12 \\
90 & 0.29 & 11.4 & 10.8 & 0.21 & 0.13 & 0.15 & 38 & 14 \\
\hline \hline
\end{tabular}

$$
\rho_{\alpha \alpha}(r)=\langle\alpha(x) \alpha(x+r)\rangle /\left\langle\alpha^{2}(x)\right\rangle .
$$

We have also estimated the integral scale $L_{q}$ associated with $\left\langle q^{2}\right\rangle$, defined such that

$$
L_{q}=\int_{0}^{r_{0}}(\langle u(x) u(x+r)\rangle+2 v(x) v(x+r)) /\left\langle q^{2}(x)\right\rangle \mathrm{d} r,
$$

where $r_{0}$ is the location of the first zero-crossing of the integrand. The ratio $u^{\prime} / v^{\prime}$, which quantifies the large scale anisotropy, is 1.25 on average. Hussein, Capp, and George ${ }^{15}$ and Panchapakesan and Lumley ${ }^{32}$ obtained 1.25 and 1.3, respectively. The fact that these ratios are constant beyond $x / D=30$ indicates that a relative equilibrium of these second-order quantities has been achieved on the axis. The turbulence intensity is close to $25 \%$ (compared to $22 \%$ in Ref. 15 and $24 \%$ in Ref. 32), which accounts for the large errors associated with the use of Taylor's hypothesis. ${ }^{28}$ The average value of $L_{u} / L_{v}$ is close to 1.75 , on average, smaller than the isotropic value of 2 . For comparison, the grid turbulence of Ref. 22, at a distance $x=42 M$ ( $M$ is the grid mesh size) yields $u^{\prime} / v^{\prime} \simeq 1.17$ and $L_{u} / L_{v} \simeq 2.6$, but with much lower turbulence intensity $\left(u^{\prime} / U \simeq 0.025\right)$. Detailed tabulations of some of the statistical quantities connected with the velocity and length scales are given in Tables III-V.

The radial profiles of $U$ (Fig. 4) collapse approximately, when normalized by the local half-velocity radius $\left(R_{0.5}\right)$. Satisfactory collapse is also exhibited by the fluctuating intensity profiles, Fig. 5. The normalized distributions of $\left\langle q^{2}\right\rangle$ appear to comply with similarity more satisfactorily than those

TABLE V. Dissipation rate coefficient and related length scales at three streamwise locations.

\begin{tabular}{cccccc}
\hline \hline $\begin{array}{c}x / D \\
-\end{array}$ & $C_{\varepsilon_{\text {iso }}}$ & $C_{\varepsilon_{\text {hom }}}$ & $\begin{array}{c}R_{0.5} \\
(\mathrm{~m})\end{array}$ & $\begin{array}{c}\ell=\left\langle q^{2}\right\rangle^{3 / 2} /\langle\varepsilon\rangle_{\text {hom }} \\
(\mathrm{m})\end{array}$ & $\begin{array}{c}\ell=u^{\prime 3} /\langle\varepsilon\rangle_{\text {iso }} \\
(\mathrm{m})\end{array}$ \\
\hline 40 & 1.65 & 1.10 & 0.26 & 0.72 & 0.16 \\
60 & 1.79 & 1.16 & 0.43 & 1.11 & 0.24 \\
80 & 2.02 & 1.16 & 0.57 & 1.47 & 0.28 \\
\hline \hline
\end{tabular}




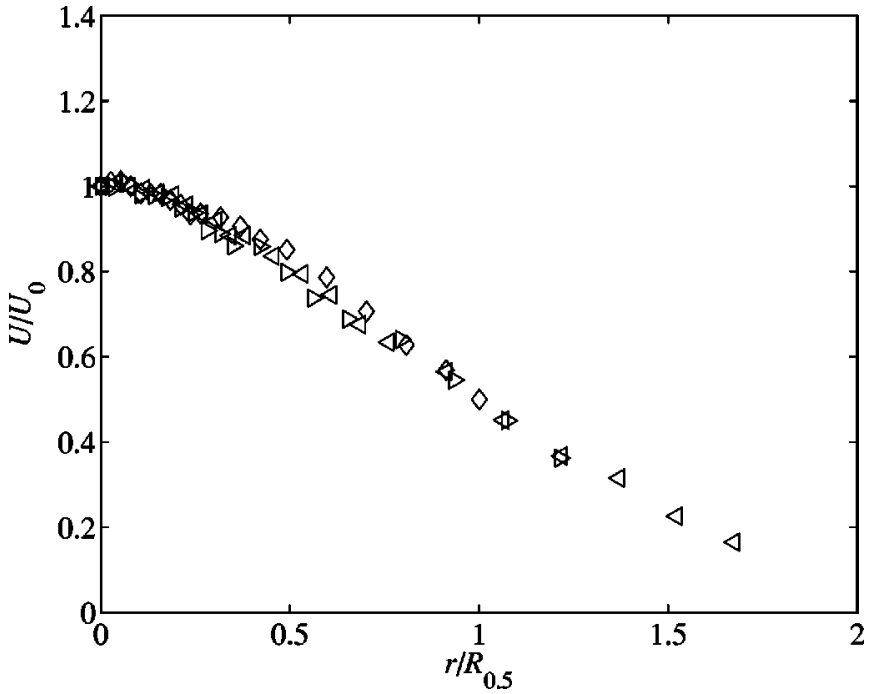

FIG. 4. Radial profiles of the streamwise velocity at three axial locations: $\triangleleft$, $x / D=40 ; \nabla, x / D=60 ; \diamond, x / D=80 . R_{0.5}$ is the half-velocity radius.

of $\left\langle u^{2}\right\rangle$. This seems to be due to a reciprocal compensation of the fluctuation intensities of $u$ and $v$, leading to a more uniform distribution of the mean energy.

The statistics of $(\partial u / \partial x)$ and $(\partial v / \partial x)$, Table III, can be used to characterize the behavior of the small scales. As noted earlier, these spatial derivatives are inferred from time derivatives, by invoking MTH. The first of these derivatives is used to define the more conventional Taylor microscale $\lambda\left(\equiv u^{\prime} /(\partial u / \partial x)^{\prime}\right)$, which combines some of the large scale properties, through $u^{\prime}$, with the characteristics of the smallest scales, via the velocity derivative. The variation of $R_{\lambda}$ $=\lambda u^{\prime} / \nu$ along the jet axis is plotted in Fig. $6 . R_{\lambda}$ is approximately constant, with a mean value of about 450 , and nearly all the values are within the experimental uncertainty (for $x / D<40$, the similarity of the small scales statistics appears not to be achieved yet, in agreement with the suggestion in

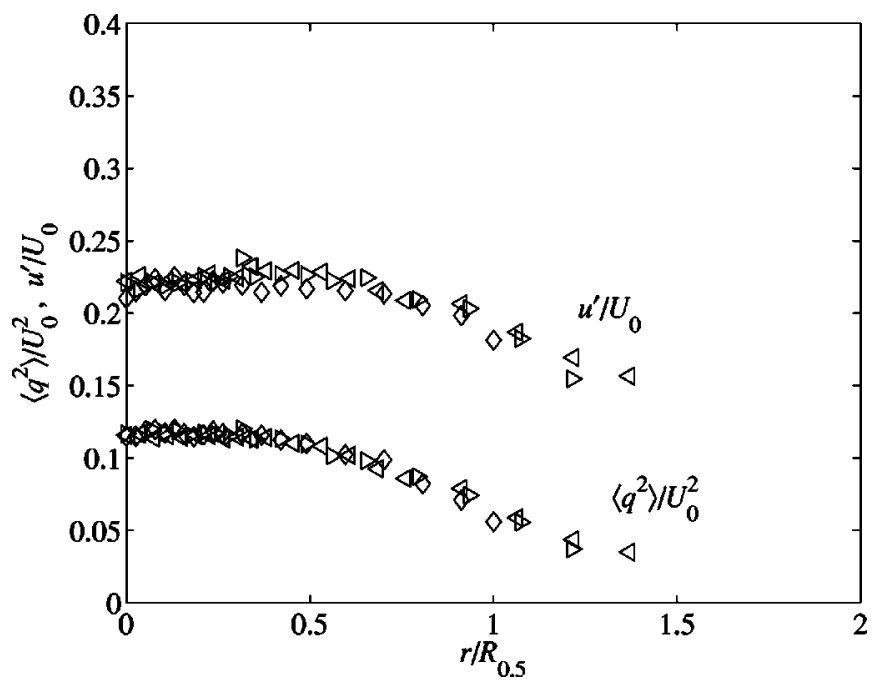

FIG. 5. Radial profiles of the streamwise velocity fluctuations intensities at three axial locations: $\triangleleft, x / D=40 ; \nabla, x / D=60 ; \diamond, x / D=80 . R_{0.5}$ is the half-velocity radius.

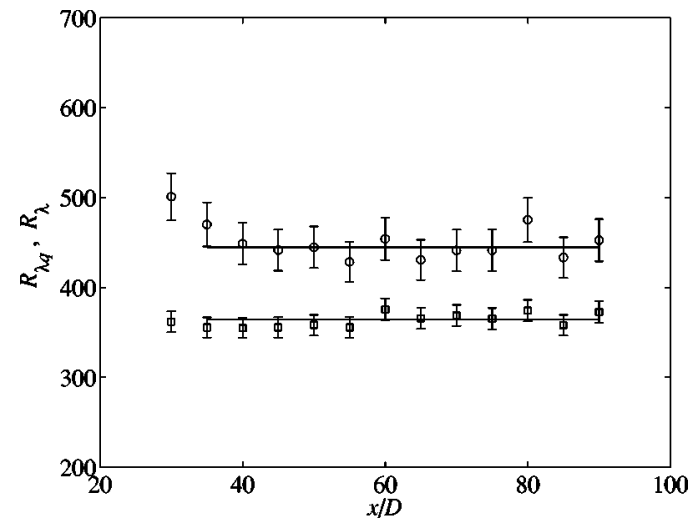

FIG. 6. Axial distribution of $R_{\lambda}(\bigcirc)$ and $R_{\lambda q}(\square)$ with error bars. Horizontal lines represent average values over the range $35 \leqslant x / D \leqslant 90$.

Ref. 8). This constancy is in accord with the circular jet results of Antonia et al. ${ }^{33}$

The mean energy dissipation rate, estimated from isotropy, is given by

$$
\langle\epsilon\rangle_{\text {iso }}=15 \nu\left\langle\left(\frac{\partial u}{\partial x}\right)^{2}\right\rangle,
$$

or, in spectral form,

$$
\langle\epsilon\rangle_{\text {iso }}=15 \nu \int_{0}^{\infty} k_{1}^{2} \phi_{u}\left(k_{1}\right) d k_{1},
$$

where the $1 \mathrm{D}$ energy spectral density $\phi_{u}\left(k_{1}\right)$ is defined such that

$$
\int_{0}^{\infty} \phi_{u}\left(k_{1}\right) d k_{1}=u^{\prime 2} .
$$

This normalization is global in nature, meaning that it constrains the area under to spectrum to a given value, and has to be applied to the spectrum calculated from a signal of suitable duration to ensure that the velocity variance is estimated correctly. (If the original signal is broken up into constant width windows, a common practice for reducing the scatter in the Fourier transform, a significant part of the energy at low frequencies may be lost.) Subsequently, the Kolmogorov length and velocity scales are defined by $\eta$ $=\left(\nu^{3} /\langle\epsilon\rangle_{\text {iso }}\right)^{1 / 4}, u_{K}=\left(\langle\epsilon\rangle_{\text {iso }} \nu\right)^{1 / 4}$, respectively. A homogeneous expression for the mean energy dissipation rate is

$$
\langle\epsilon\rangle_{\mathrm{hom}}=3 \nu\left[\left\langle\left(\frac{\partial u}{\partial x}\right)^{2}\right\rangle+2\left\langle\left(\frac{\partial v}{\partial x}\right)^{2}\right\rangle\right] .
$$

This departure from local isotropy seems consistent with the observation by George and Hussein ${ }^{34}$ that, on the axis of the jet, local axisymmetry provided a more reliable estimate of $\langle\epsilon\rangle$ than local isotropy. Having introduced $\langle\epsilon\rangle_{\text {hom }}$, a more general definition of the Taylor microscale would be $\lambda_{q}$ $=\left(5 \nu\left\langle q^{2}\right\rangle /\langle\epsilon\rangle_{\text {hom }}\right)^{1 / 2}{ }^{6}$ The corresponding turbulence Reynolds number is $R_{\lambda q} \equiv\left(\left\langle q^{2}\right\rangle / 3\right)^{1 / 2} \lambda_{q} / \nu$, which is also included in Fig. 6. The magnitude of $R_{\lambda q}$ is smaller than that of $R_{\lambda}$, because the energy dissipation rate estimated using $\left\langle(\partial v / \partial x)^{2}\right\rangle$ falls short of the isotropic value and, thus, reduces the estimate of $\langle\epsilon\rangle_{\text {hom }}$ with respect to $\langle\epsilon\rangle_{\text {iso. }}$. The scatter in the 


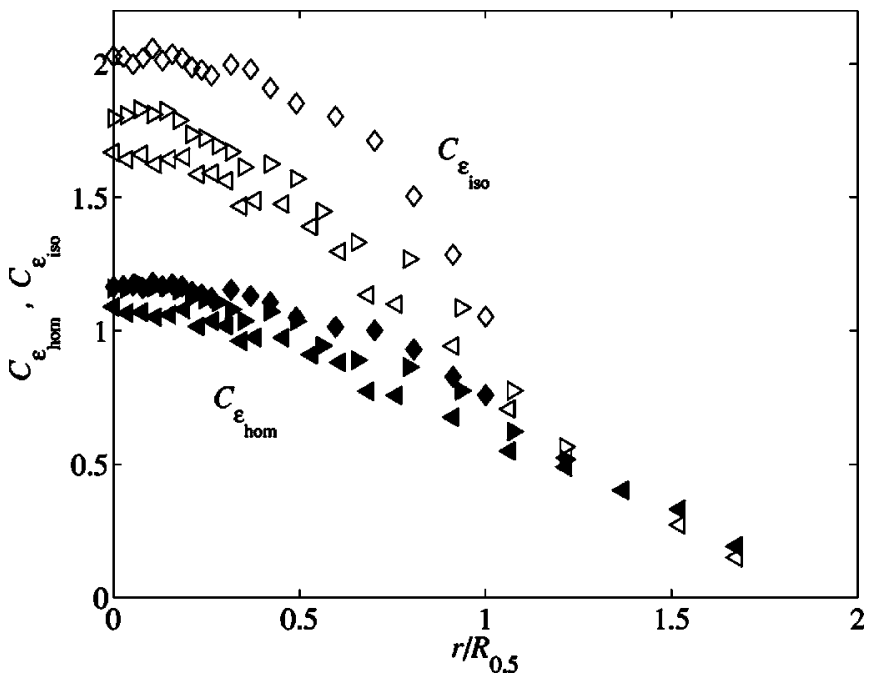

FIG. 7. Radial variation of $C_{\epsilon_{\text {hom }}}\left(\equiv 3 R_{0.5}\langle\epsilon\rangle_{\text {hom }} /\left\langle q^{2}\right\rangle^{3 / 2}\right)$, filled symbols, and $\left(C_{\epsilon_{\text {iso }}} \equiv R_{0.5}\langle\epsilon\rangle_{\text {iso }} / u^{\prime 3}\right)$, empty symbols: $\triangleleft, \varangle x / D=40 ; \triangleright, \nabla, x / D=60 ; \diamond$, $\checkmark, x / D=80$. In these definitions the axial values of the $\left\langle q^{2}\right\rangle$ and $u^{\prime 2}$ were used, respectively.

values of $R_{\lambda q}$ is also reduced to $2.7 \%$, and now all the points for $x / D \geqslant 35$ have the same value, to within the experimental uncertainty. The reduced scatter in $R_{\lambda q}$ reflects also the higher uniformity in $x$ of $\left\langle q^{2}\right\rangle$ compared to $\left\langle u^{2}\right\rangle$.

Even though in this paper we focus on the axial development of the flow, it is interesting to have a brief look at the radial profiles of some quantities. Estimates of $\ell$, defined as $\langle\epsilon\rangle_{\text {hom }} /\left\langle q^{2}\right\rangle^{3 / 2}$, and the half-velocity radius $R_{0.5}$ are evaluated for three axial stations $(x / D=[40,60,80])$, Table $\mathrm{V}$. The nondimensional coefficient $C_{\epsilon_{\mathrm{hom}}} \equiv R_{0.5}\langle\epsilon\rangle_{\mathrm{hom}} /\left\langle q^{2}\right\rangle^{3 / 2}$ is approximately constant unlike $C_{\epsilon_{\text {ise }}} \equiv R_{0.5}\langle\epsilon\rangle_{\text {iso }} / u^{\prime 3}$, which substantially increases with $x$. There is little doubt that the use of $\langle\epsilon\rangle_{\text {hom }}$ leads to an improvement in the similarity of radial profiles of the energy dissipation rate, Fig. 7.

The departure from isotropy of the small scales can be quantified by the ratio $I=\left\langle(\partial v / \partial x)^{2}\right\rangle /\left\langle(\partial u / \partial x)^{2}\right\rangle$, Table III: the average value of $I$ is 1.7 , in the range $35 \leqslant x / D \leqslant 90$. In comparison, for grid turbulence, $I$ is typically 1.8 , slightly closer to the isotropic value of 2 . Champagne, ${ }^{35}$ who also applied corrections for Taylor's hypothesis in high turbulence intensity, reported a value of $I$ of 1.6 at $x / D=70$ for a round jet with $R_{\lambda}=626$.

\section{ENERGY SPECTRA AND STRUCTURE FUNCTIONS}

Having examined the analytical conditions for similarity of the energy structure functions in Sec. III, we now proceed to the experimental verification of these conditions in the far-field of the jet. The relation between $\phi_{u}\left(k_{1}\right)$ and $\left\langle(\delta u)^{2}\right\rangle$, viz.

$$
\left\langle(\delta u)^{2}\right\rangle=2 \int_{0}^{\infty} \phi_{u}\left(k_{1}\right)\left[1-\cos \left(k_{1} r\right)\right] \mathrm{d} k_{1},
$$

allows $\left\langle(\delta u)^{2}\right\rangle$ to be inferred from $\phi_{u}\left(k_{1}\right)$. The transformation from the spectrum to the structure function involves an integration and should, therefore, produce an output which is

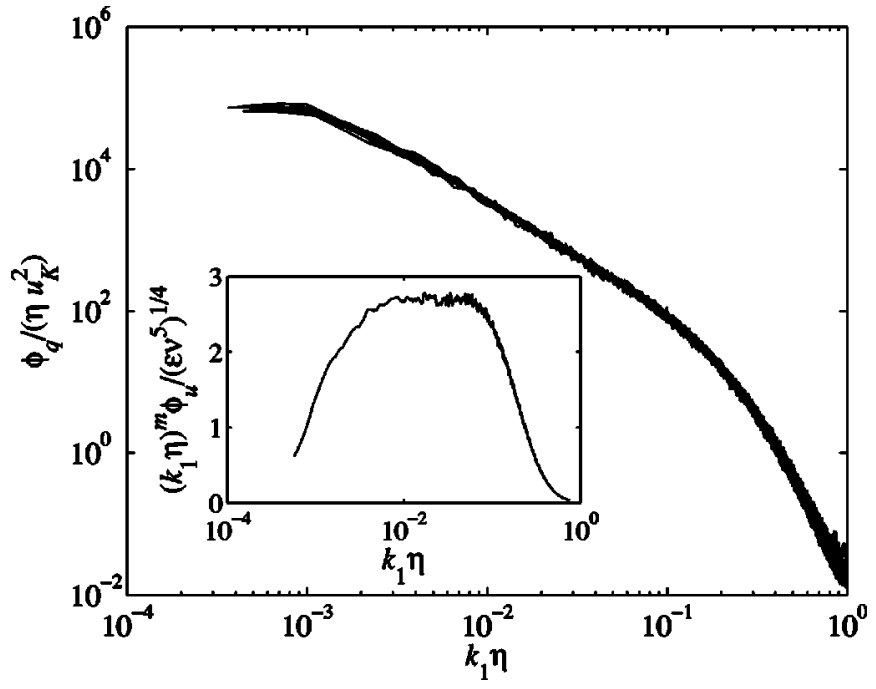

FIG. 8. Spectra of the turbulent energy $\left\langle q^{2}\right\rangle$ in the range $35 \leqslant x / D \leqslant 90$ normalized by Kolmogorov scales. The inset reports the compensated spectrum (with $m=-1.52$ ) in semi-logarithmic scale, after averaging over the axial stations, highlighting the inertial range power law.

more regular that the integrand. Because of the integral in Eq. (28), the scaling range in the physical space should be narrower than in the spectral domain. ${ }^{36,37}$ We prefer to present both spectra and structure functions, primarily to crosscheck our conclusions, but also because of the likely differences between these two quantities, which could lead to possibly different interpretations. For example, electronic noise contamination is more evident in the high wave number portion of the spectrum than at small separations in the structure functions.

Differences between spectra and structure functions may also arise because of different types of normalization they are subjected to. The power spectrum satisfies an integral constraint [Eq. (26) for $u$ and a corresponding one for $v$ ], while the structure functions converge to local (pointwise) limits, at large separations, e.g.,

$$
\begin{aligned}
& \lim _{r \rightarrow \infty} \frac{\left\langle(\delta u)^{2}\right\rangle}{u_{K}^{2}}=\frac{2\left\langle u^{2}\right\rangle}{u_{K}^{2}}=\frac{2}{15^{1 / 2}} R_{\lambda}, \\
& \lim _{r \rightarrow \infty} \frac{\left\langle(\delta v)^{2}\right\rangle}{u_{K}^{2}}=\frac{2\left\langle v^{2}\right\rangle}{u_{K}^{2}}=\frac{2}{15^{1 / 2}} \frac{\left\langle v^{2}\right\rangle}{\left\langle u^{2}\right\rangle} R_{\lambda}, \\
& \lim _{r \rightarrow \infty} \frac{\left\langle(\delta q)^{2}\right\rangle}{u_{K}^{2}}=\frac{2\left\langle u^{2}\right\rangle}{u_{K}^{2}}+2 \frac{2\left\langle v^{2}\right\rangle}{u_{K}^{2}}=\frac{2}{15^{1 / 2}}\left(1+2 \frac{\left\langle v^{2}\right\rangle}{\left\langle u^{2}\right\rangle}\right) R_{\lambda} .
\end{aligned}
$$

In the far field of the jet, these limiting values should not depend on $x$, since $R_{\lambda}$ (or $R_{\lambda q}$ ) and the ratio $\left\langle v^{2}\right\rangle /\left\langle u^{2}\right\rangle$ are constant (e.g., Figs. 3 and 6).

\section{A. Spectra}

Figures 8-10 display the distributions of 


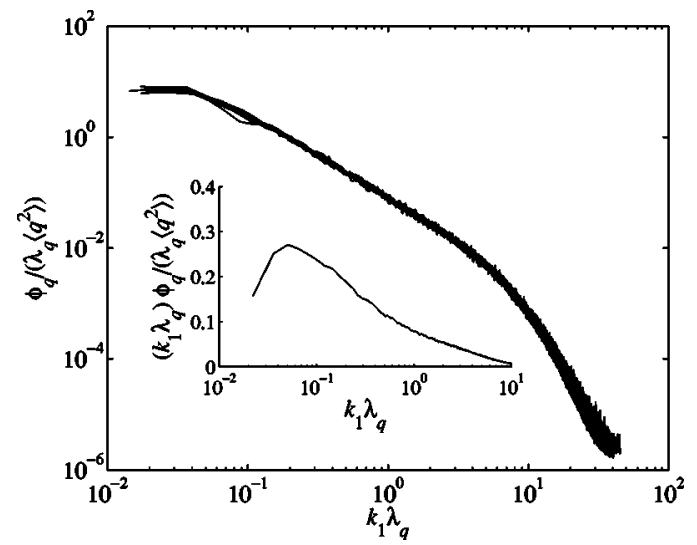

FIG. 9. Spectra of the turbulent energy $\left\langle q^{2}\right\rangle$ normalized by the Taylor microscale $\lambda_{q}$ and the turbulent energy $\left\langle q^{2}\right\rangle$. The inset shows the distribution of $\phi_{q}$ normalized such that the area under the curve represents the mean energy $\left\langle q^{2}\right\rangle$.

$$
\phi_{q}\left(k_{1}\right) \equiv \phi_{u}\left(k_{1}\right)+2 \phi_{v}\left(k_{1}\right),
$$

normalized using three main types of normalizations (as summarized in Table VI). One of these is based on the Kolmogorov scales $\eta$ and $u_{K}$. The other two use the rms velocity $\left\langle\alpha^{2}\right\rangle^{1 / 2}(\alpha \equiv\{u, v, q\})$ and either the Taylor microscale $\lambda$ or the integral length scale $L_{\alpha}$ [defined as in Eqs. (24) and (25)]. The interest of using $\phi_{q}$, (or $\left.\left\langle(\delta q)^{2}\right\rangle\right)$, hinges on the analogy between the energy and the scalar structure functions, ${ }^{38}$ as pointed out in Ref. 20 in the context of the scale-by-scale budget for the circular jet.

In general, from these figures, it appears that the collapse is adequate over the wave number range, for each choice of scaling parameters pairs, as might have been expected from the constancy of $R_{\lambda}$. The relatively wide range of spatial locations used here, $35 \leqslant x / D \leqslant 90$, reinforces this conclusion. However, a close look at the spectra reveals some systematic trends. When the integral length scale is used, Fig. 10, the spectra appear to come together more closely at relatively small wave numbers. With the Kolmogorov normalization (Fig. 8), this tendency holds at relatively large wave numbers. The normalization based on $\lambda_{q}$ and $\left\langle\alpha^{2}\right\rangle$, Fig. 9, appears to provide a satisfactorily collapse for nearly all wave numbers. (Note that, at very large wave numbers, the spectral density begins to increase. This upturn is clearly not physical and is due to electronic noise, which is unavoidable in this type of experiment. Spectra were not

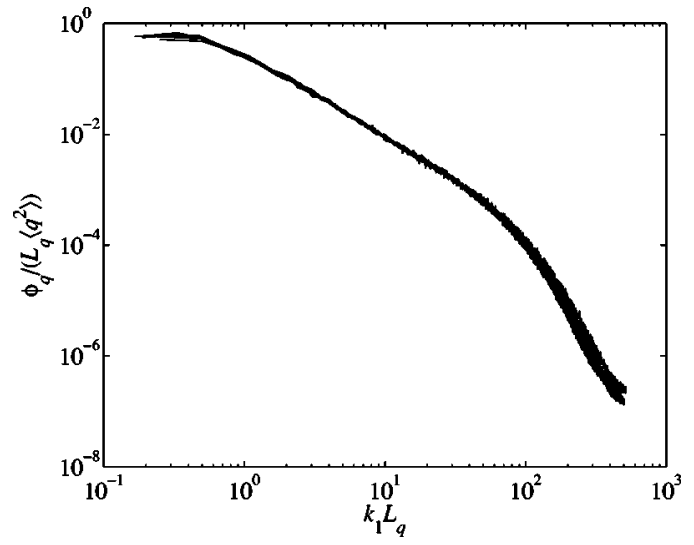

FIG. 10. Spectra of the turbulent energy $\left\langle q^{2}\right\rangle$ normalized by $L_{q}$ and $\left\langle q^{2}\right\rangle$.

corrected for this noise because of the subjectivity involved in this procedure.) The level of collapse of the spectra was quantified directly by calculating the dispersion (viz. the rms) of the spectral values at every wave number. The dispersion is defined as

$$
\sigma_{\mathrm{SP}}=\frac{\left[\widetilde{\phi}_{q}(\widetilde{k})\right]^{\prime}}{\widetilde{\widetilde{\phi}_{q}(\widetilde{k})}} .
$$

Here, the tilde denotes normalization according to any of the chosen parametric pairs and the overbar the average of the spectral density data (at the same wave number) for all the locations on the jet axis (in the range $35 \leqslant x / D \leqslant 90$ ). Values of $\sigma_{\mathrm{SP}}$ are shown in Fig. 11, where the abscissa represents the wave number divided by the maximum measured wave number, which corresponds quite closely to the Kolmogorov scale, at all the axial locations. Even though the curves are jittery (the spectra exhibit intrinsic scatter), it appears that at low wave numbers - corresponding to values of the length scale around $L_{q}$ - the dispersion is generally lowest when the normalization is based on $L_{q}$ whereas, at high wave numbers, the Kolmogorov-based scaling yields the lowest values of $\sigma_{\mathrm{SP}}$. When the scaling is based on $\lambda_{q}$, the dispersion falls roughly between the two previous estimates, but a precise wave number, for which either the $L_{q}$-based or Kolmogorovbased normalization is more pertinent, is not clearly discernible. In addition, it is also apparent that the dispersion increases at large wave numbers, regardless of the choice of

TABLE VI. Scales for the normalization of spectra and structure functions (results for pairs in squared brackets

\begin{tabular}{|c|c|c|c|c|}
\hline \multirow[b]{2}{*}{ Length scales } & \multicolumn{2}{|c|}{ Spectra } & \multicolumn{2}{|c|}{ Structure functions } \\
\hline & $\cdot=\phi_{\alpha}$ & $\cdot=\phi_{q}$ & $\cdot=\left\langle(\delta \alpha)^{2}\right\rangle$ & $\cdot=\left\langle(\delta q)^{2}\right\rangle$ \\
\hline$\eta$ & {$\left[k \eta, \bullet / \eta u_{K}^{2}\right]$} & $k \eta, \bullet / \eta u_{K}^{2}$ & {$\left[r / \eta, \bullet / u_{K}^{2}\right]$} & $r / \eta, \bullet / u_{K}^{2}$ \\
\hline$\lambda$ & {$\left[k \lambda, \bullet / \lambda \alpha^{\prime 2}\right]$} & {$\left[k \lambda, \bullet / \lambda\left\langle q^{2}\right\rangle\right]$} & {$\left[r / \lambda, \bullet / \alpha^{\prime 2}\right]$} & {$\left[r / \lambda, \bullet /\left\langle q^{2}\right\rangle\right]$} \\
\hline$\lambda_{q}$ & {$\left[k \lambda_{q}, \bullet / \lambda_{q} \alpha^{\prime 2}\right]$} & $k \lambda_{q}, \bullet / \lambda_{q}\left\langle q^{2}\right\rangle$ & {$\left[r / \lambda_{q}, \bullet / \alpha^{\prime 2}\right]$} & $r / \lambda_{q}, \bullet /\left\langle q^{2}\right\rangle$ \\
\hline$L_{u}, L_{v}$ & {$\left[k L_{\alpha}, \bullet / L_{\alpha} \alpha^{\prime 2}\right]$} & - & {$\left[r / L_{\alpha}, \bullet / \alpha^{\prime 2}\right]$} & - \\
\hline$L_{q}$ & {$\left[k L_{q}, \bullet / L_{q}\left\langle q^{2}\right\rangle\right]$} & $k L_{q}, \bullet / L_{q}\left\langle q^{2}\right\rangle$ & {$\left[r / L_{q}, \bullet /\left\langle q^{2}\right\rangle\right]$} & $r / L_{q}, \bullet /\left\langle q^{2}\right\rangle$ \\
\hline
\end{tabular}
have not been shown here). 


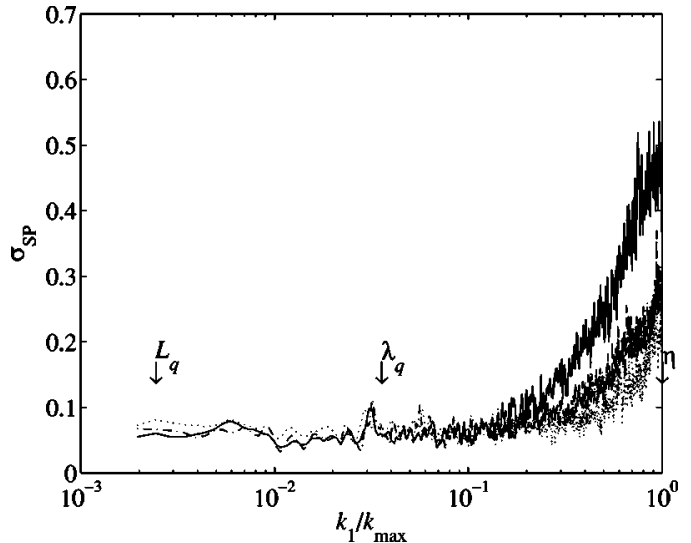

FIG. 11. Relative dispersion $\left[\sigma_{\mathrm{SP}}\right.$, defined by Eq. (32)] of the spectra at different $x$ locations as a function of $k_{1}$. The three curves correspond to the three different normalizations used in Fig. 8-10. Normalization based on: —, integral length scale and $\left\langle q^{2}\right\rangle ; \cdot---$, Taylor microscale and $\left\langle q^{2}\right\rangle ; \cdots$, Kolmogorov scales. $k_{\max }$ corresponds to the largest wave number for which the spectra have been calculated.

scaling parameters. This seems to be caused by the contribution from electronic noise.

The compensated distribution of $\phi_{q}$ (inset of Fig. 8) highlights that there is a significant range of scales where the spectrum follows a power law. Over this range, a least squares fit to the spectrum yields a slope of $m=-1.52$, compared to the prediction of -1.67 of Kolmogorov ${ }^{18}$ and Obukhov, ${ }^{39}$ the difference being likely produced by the finite value of $R_{\lambda}{ }^{40-42}$ Such an ad hoc compensation of the spectrum does not highlight the spectral "bump" at the high-wave number end of the scaling range, ${ }^{43}$ although this feature would become noticeable when the spectrum is multiplied by $\left(k_{1} \eta\right)^{5 / 3}$.

The inset in Fig. 9 shows that the most energetic scale is located at $k \lambda_{q} \simeq 0.05$. This provides an estimate of the integral length scale, which is indeed nearly 20 times larger than $\lambda_{q}$ (see Table IV). By comparison, for active grid turbulence at $R_{\lambda} \simeq 262$, the peak in the energy spectrum is at $k \simeq 0.1,{ }^{40}$ which corresponds to an integral scale of $0.1 \mathrm{~m}$ (see their Table I), while the Taylor microscale is $\simeq 16$ times smaller. The larger separation between $\lambda_{q}$ and $L_{q}$ in the jet reflects the higher Reynolds number, since one expects $L / \lambda$ to be proportional to $R_{\lambda}$ (if local isotropy is assumed).

George ${ }^{1,4}$ showed that, for decaying homogeneous and isotropic turbulence, $\lambda$ and $\left\langle q^{2}\right\rangle^{1 / 2}$ are scales which allow the spectral energy equation to admit to similarity. Support for this was provided by grid turbulence measurements of Comte-Bellot and Corrsin. ${ }^{44}$ The grid turbulence data of Antonia et al. ${ }^{6}$ also provided support for $\lambda$ and $\left\langle q^{2}\right\rangle^{1 / 2}$. However, these authors also found that the Kolmogorov variables $u_{K}$ and $\eta$ collapsed the high wave number part of $\phi_{q}$ satisfactorily, at least as well as a $\lambda$-based normalization. As there was no attempt to quantify the degree of collapse, it was not possible to assess unambiguously the relative merits of these two normalizations at large $k_{1}$; the difficulty was possibly compounded by the insufficient spatial resolution of the hot wires. Recently, using well resolved DNS data for low Reynolds number decaying turbulence in a periodic box, Antonia

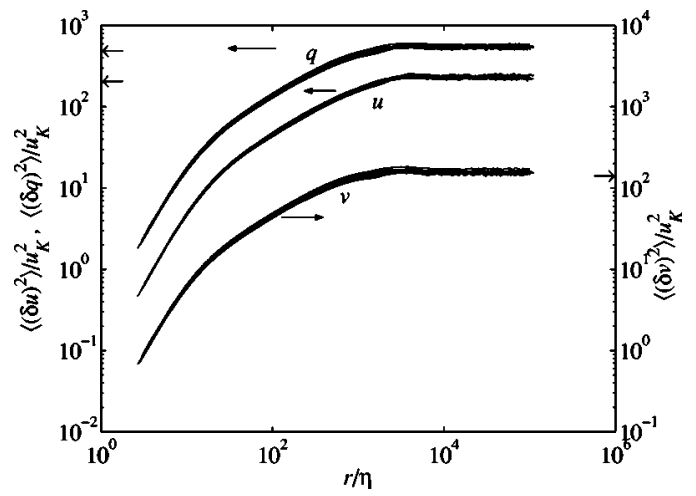

FIG. 12. Structure functions of $u, v$, and $q$ normalized by Kolmogorov scales. Arrows against the two ordinates indicate limiting values, averaged over the axial locations in the range $35 \leqslant x / D \leqslant 90$, according to Eqs. (29)-(31).

and Orlandi ${ }^{7}$ found that for sufficiently large $k$, normalization based on $u_{K}$ and $\eta$ provided a better collapse of $E(k)$ and $T(k)$ - the 3D energy spectrum and nonlinear energy transfer function, respectively-than a normalization based on $\left\langle q^{2}\right\rangle^{1 / 2}$ and $\lambda$.

\section{B. Structure functions}

Structure functions of $q^{2}$, normalized according to the parametric groupings set out in Table VI, are represented in Figs. 12-14. Previous comments on the relative behaviors of the spectra for the three types of normalization considered, also apply in general to the structure functions. These curves are, however, smoother, compared to the spectra, hence the discrepancies between different normalizations are less ambiguous. From these figures it can be inferred that the Taylorbased normalization performs adequately for almost all separations. Nonetheless, at small separations, $u_{K}$ and $\eta$ provide a better quality of collapse of the structure functions. Finally, when the integral length scale $L_{q}$ and $\left\langle q^{2}\right\rangle^{1 / 2}$ are used, the collapse at large separations appears to be improved. In order to quantify this improvement, we estimate once more the dispersion, defined, analogously to Eq. (32), as the rootmean squared (rms) value of $\left\langle(\delta q)^{2}\right\rangle$ (at every value of $r$ ) for

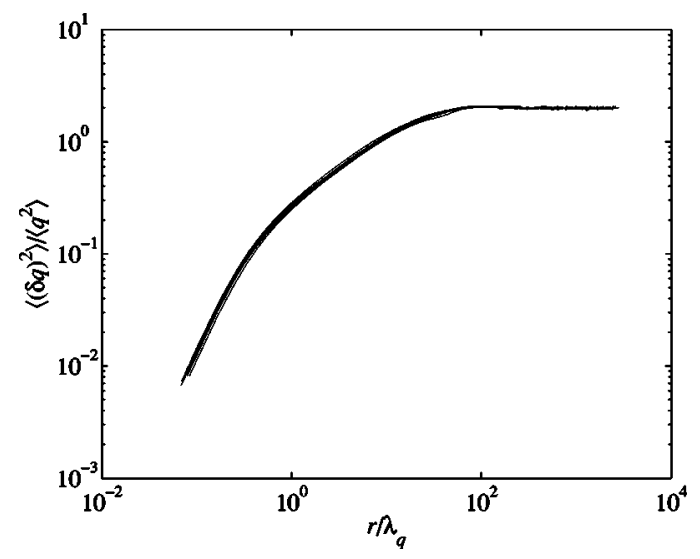

FIG. 13. Structure functions of the mean energy normalized by the Taylor microscale $\lambda_{q}$ and the turbulent energy $\left\langle q^{2}\right\rangle$. 


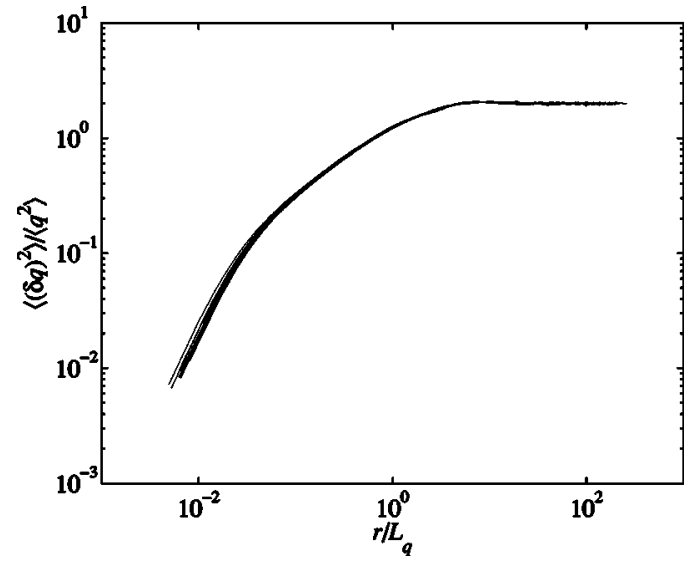

FIG. 14. Structure functions of the mean energy normalized by the integral length scale $L_{q}$ and the turbulent energy $\left\langle q^{2}\right\rangle$.

all the values of $x / D$, normalized by the mean value (indicated by the overbar) of $\left\langle(\delta q)^{2}\right\rangle$ over different axial stations, i.e.,

$$
\sigma_{\mathrm{SF}}=\frac{\left[\left\langle(\delta q)^{2}\right\rangle\right]^{\prime}}{\overline{\left\langle(\delta q)^{2}\right\rangle}}
$$

The result is plotted in Fig. 15 in terms of the separation $r$ normalized by the largest separation used in calculating the structure functions. As was observed for the spectra, the smallest values of $\sigma_{\mathrm{SF}}$ at small $r$ correspond to the Kolmogorov normalization. At large separations, $\sigma_{\mathrm{SF}}$ is smallest for the normalization based on $L_{q}$ and $\left\langle q^{2}\right\rangle^{1 / 2}$. The differences between the three curves for $\sigma_{\mathrm{SF}}$ are relatively larger at small $r$ than large $r$. In the latter case, the undulations in $\sigma_{\mathrm{SF}}$ are apparently linked to the variations in the limiting valuesEqs. (29)-(31) — which reflect the finite duration of the velocity records. A Taylor-based normalization provides an adequate overall collapse, since the corresponding values of $\sigma_{\mathrm{SF}}$ lie between those for the other two normalizations for $\log (r) / \log \left(r_{\max }\right) \leqq 0.7$, attaining comparable values to the integral normalization for larger separations. Interestingly, the crossover between the $\eta$-based and $L_{q}$-based normalizations

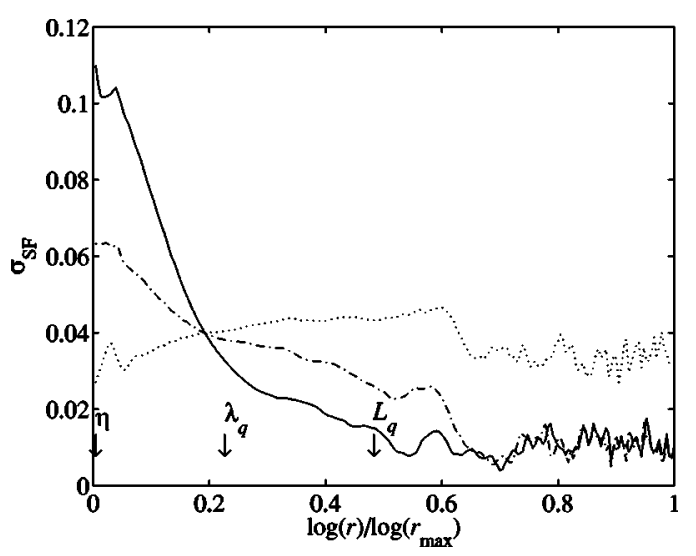

FIG. 15. Relative dispersion $\left[\sigma_{\mathrm{SF}}\right.$, defined by Eq. (33)] for the structure functions normalized in three different ways. $r_{\max }$ corresponds to the largest separation for which the structure functions have been calculated. Normalization based on: -, integral scale; ----, Taylor scale; $\cdots$, Kolmogorov scale. is close to $\lambda_{q}$. It would seem that the Taylor microscale corresponds to the scale where the large and small scales normalizations exchange roles in terms of collapsing the structure functions. This feature could be taken into account in devising an empirical parametric model of the structure functions.

The limiting values of $\left\langle(\delta \alpha)^{2}\right\rangle / u_{K}^{2}$, calculated assuming local isotropy in Eqs. (29) and (30), are reported in Fig. 12, as indicated by the open arrows. The values are 222 for $u$ and 147 for $v$, approximately $5 \%$ lower than the measured values (233 for $u$ and 155 for $v$ ). In comparison, for twodimensional wakes behind cylinders, the limit for $u$ displays the same level of accuracy, while that for $v$ has a marked dependence on the type of wake generator. ${ }^{3}$ The limit of $\left\langle(\delta q)^{2}\right\rangle / u_{K}^{2}$, as estimated using Eq. (31), is also reported in the same figure: Its value is 522 , viz. nearly $4 \%$ smaller than the average measured value (544).

The approach towards the limiting values of $\left\langle(\delta \alpha)^{2}\right\rangle$ as $r \rightarrow \infty$ is achieved in a monotonic manner, i.e., there is no marked periodicity in the flow and the role of the large coherent structures may be very limited from an energy viewpoint. An opposite trend was observed in the cylinder wakes examined by Antonia et al. $;^{3}$ there, the structure functions of the lateral velocity component exhibited a clear overshoot before returning to their limiting value (accordingly, there was a distinct peak in the spectrum). This behavior reflects the persisting action of the vortices shed by the wake generators. Speculatively, for the $2 \mathrm{D}$ wake, for which $R_{\lambda}$ is also constant along $x$, the similarity of structure functions and spectra may be less satisfactory than for the jet (this point needs to be investigated in a future research). Coherent structures in the far field of the jet have been investigated by means of phase averaging by Tso and Hussain ${ }^{45}$ but the energy content of the most probable helical structures remains difficult to assess directly. ${ }^{46}$ The success of the scaling of the structure functions and spectra found here is compatible with a picture where the coherent structures do not play a dominant role. A connection between the scaling of one-point statistics and the organization of the flow has been recently investigated in Ref. 47 where it was shown that the collapse of the moments (up to order three) of velocity fluctuations in a shear layer is improved when the coherent structures are inhibited.

\section{CONCLUSIONS}

Traditional arguments indicate that mean velocity and Reynolds stress profiles in a round jet should exhibit a selfsimilar behavior. However, the influence of the initial conditions or Reynolds number on this similarity state cannot be ruled out, as indicated by George. ${ }^{1}$ Many analyses of single point statistical moments (i.e., those related by the Reynolds averaged equations) have been reported for the round jet in previous investigations. We have preferred to focus on the two-point statistics - that is, involving the difference of the velocity at points separated in time, converted to a spatial separation via Taylor's hypothesis—and have applied a similarity analysis to the transport equation for structure functions. This analysis indicates that the Taylor microscale $\lambda$ is 
the relevant length scale. However, since the turbulence Reynolds number remains constant with $x$, the Kolmogorov microscale $\eta$, the Taylor microscale $\lambda$ and the integral length scale $L$ should develop with $x$ proportionately to each other. The constancy of $R_{\lambda}$ suggests that the degree with which structure functions and spectra comply with similarity should be equally satisfactory, regardless of the choice of scaling parameters. From an analytical point of view, the round jet should exhibit similarity across the complete range of length scales.

The existence of equilibrium similarity has not been rigorously demonstrated. It has only been assumed, as for the Blasius solution of a laminar boundary layer. A formal justification of the present solution is not apparent. Experimental corroboration of the similarity analysis is thus of fundamental importance. Experimental data in the far field of the jet $(35 \leqslant x / D \leqslant 90)$, after correcting for the effects of spatial attenuation of the X-wire and/or Taylor's hypothesis, support the conclusion that the three types of normalization collapse the spectra and structure functions reasonably well. The $\lambda$-based scaling is satisfactory over nearly the whole range of scales, although, at length scales of the order of $\eta$ and $L$, Kolmogorov-based and integral-based normalizations are superior in the sense that the corresponding collapses in these ranges exhibit smaller dispersions. An intuitive explanation for the adequate overall performance of the $\lambda$-based scaling may be linked to the definition of this scale; it combines information from large scales (via $\left\langle q^{2}\right\rangle$ ) and small scales (through the dissipation rate). It is conceivable, although difficult to verify experimentally, that in the limit of very large Reynolds numbers and with adequately resolved measurements of the smallest and largest scales, the three scalings will yield an equally good collapse of the spectra and structure functions. Further verification of these ideas is more likely to occur for a jet flow, compared to (static) grid turbulence, because of the relatively high values of $R_{\lambda}$ that can be achieved and the absence of boundaries. (Active grids can achieve comparable or larger values of $R_{\lambda}$, somehow at the expense of the large scale inhomogeneity, due to the forcing that is applied to the flow.) A natural outcome of the relevance of the Taylor-based normalization would be the development of a general parametric model for the structure function or the spectrum. At present, many of the models commonly used to describe the spectrum (or structure function) are parametrized on the values of these functions at small and large separations (e.g., Ref. 48 and references therein). The slope in the inertial range is then considered separately, possibly as a function of the Reynolds number. A unifying approach, which requires less experimentally tunable parameters and hinges on the Taylor microscale, will be attempted in the future with the aim of obtaining a more general model.

\section{ACKNOWLEDGMENTS}

The support of the Australian Research Council is gratefully acknowledged. We also much appreciated the contributions by Dr. G. Xu to an earlier set of experiments carried out in the same flow.
${ }^{1}$ W. K. George, "The self-preservation of turbulent flows and its relation to initial conditions and coherent structures," in Advances in Turbulence, edited by W. K. George and R. Arndt (Springer, Berlin, 1989), pp. 39-74. ${ }^{2}$ I. Wygnanski, F. Champagne, and B. Marasli, "On the large-scale structures in two-dimensional, small-deficit, turbulent wakes," J. Fluid Mech. 168, 31 (1986).

${ }^{3}$ R. A. Antonia, T. Zhou, and G. P. Romano, "Small-scale turbulence characteristics of two-dimensional bluff body wakes," J. Fluid Mech. 459, 67 (2002).

${ }^{4}$ W. K. George, "The decay of homogeneous isotropic turbulence," Phys. Fluids A 4, 1492 (1992).

${ }^{5}$ W. K. George and M. M. Gibson, "The self-preservation of homogeneous shear flow turbulence," Exp. Fluids 13, 229 (1992).

${ }^{6}$ R. A. Antonia, R. J. Smalley, T. Zhou, F. Anselmet, and L. Danaila, "Similarity of energy structure functions in decaying homogeneous isotropic turbulence," J. Fluid Mech. 487, 245 (2003).

${ }^{7}$ R. A. Antonia and P. Orlandi, "Similarity of decaying isotropic turbulence with a passive scalar," J. Fluid Mech. 505, 123 (2004).

${ }^{8}$ H. Tennekes and J. L. Lumley, A First Course in Turbulence (MIT Press, Cambridge, MA, 1972).

${ }^{9}$ J. C. Rotta, "Turbulent boundary layers in incompressible flow," in Progress in Aeronautical Sciences, edited by A. Ferri, D. Kchemann, and L. H. G. Sterne (Pergamon, New York, 1962), Vol. 2, pp. 1-220.

${ }^{10}$ J. Mathieu and J. Scott, An Introduction to Turbulent Flow (Cambridge University Press, Cambridge, 2000).

${ }^{11}$ D. J. Tritton, Physical Fluid Dynamics, 2nd ed. (Oxford University Press, Oxford, 1988).

${ }^{12}$ B. J. Boersma, G. Brethouwer, and F. T. M. Nieuwstadt, "A numerical investigation on the effect of the inflow conditions on the self-similar region of a round jet," Phys. Fluids 10, 899 (1998).

${ }^{13}$ S. A. Stanley, S. Sarkar, and J. P. Mellado, "A study of the flow-field evolution and mixing in a planar turbulent jet using direct numerical simulation," J. Fluid Mech. 450, 377 (2002).

${ }^{14}$ A. S. Monin and A. M. Yaglom, Statistical Fluid Mechanics: Mechanics of Turbulence (MIT Press, Cambridge, MA, 1971), Vol. 1.

${ }^{15}$ H. J. Hussein, S. P. Capp, and W. K. George, "Velocity measurements in a high-Reynolds-number, momentum-conserving, axisymmetric, turbulent jet," J. Fluid Mech. 258, 31 (1994).

${ }^{16}$ D. Ewing and W. K. George, "Similarity analysis of the two-point velocity correlation tensor in a turbulent axisymmetric jet," in Turbulence, Heat and Mass Transfer 1, edited by K. Hanjalić and J. C. F. Pereira (Begell, New York, 1995), pp. 49-56.

${ }^{17}$ W. K. George, "Some new ideas for similarity of turbulent shear flows," in Turbulence, Heat and Mass Transfer 1, edited by K. Hanjalić and J. C. F. Pereira (Begell, New York, 1995), pp. 1 13-24.

${ }^{18}$ A. N. Kolmogorov, "Local structure of turbulence in an incompressible fluid for very large Reynolds numbers," Dokl. Akad. Nauk SSSR 30, 299 (1941).

${ }^{19}$ S. B. Pope, Turbulent Flows (Cambridge University Press, Cambridge, 2000).

${ }^{20}$ L. Danaila, R. A. Antonia, and P. Burattini, "Progress in studying smallscale turbulence using 'exact' two-point equations," New J. Phys. 6, 1 (2004).

${ }^{21}$ L. Danaila, F. Anselmet, T. Zhou, and R. A. Antonia, "A generalization of Yaglom's equation which accounts for the large-scale forcing in heated decaying turbulence," J. Fluid Mech. 391, 359 (1999).

${ }^{22} \mathrm{P}$. Burattini and R. A. Antonia, "The effect of different X-wire calibration schemes on some turbulence statistics," Exp. Fluids (to be published).

${ }^{23}$ R. J. Moffat, "Describing the uncertainties in experimental results," Exp. Therm. Fluid Sci. 1, 3 (1988).

${ }^{24}$ J. C. Wyngaard, "Measurement of small-scale turbulence structure with hot-wires," J. Sci. Instrum. 1, 1105 (1968).

${ }^{25}$ D. O. Martinez, S. Chen, G. D. Doolen, L.-P. Wang, and Y. Zhou, "Energy spectrum in the dissipation range of fluid turbulence," J. Plasma Phys. 57, 195 (1997).

${ }^{26}$ Y. Zhu and R. A. Antonia, "Effect of wire separation on X-probe measurements in a turbulent flow," J. Fluid Mech. 287, 199 (1995).

${ }^{27}$ J. L. Lumley, "Interpretation of time spectra measured in high-intensity shear flows," Phys. Fluids 8, 1056 (1965).

${ }^{28} \mathrm{G}$. Heskestad, "A generalized Taylor hypothesis with application for high Reynolds number turbulent shear flows," J. Appl. Mech. 32, 735 (1965).

${ }^{29}$ H. Kahalerras, Y. Malcot, Y. Gagne, and B. Castaing, "Intermittency and Reynolds number," Phys. Fluids 10, 910 (1998).

${ }^{30}$ P. Burattini, R. Antonia, S. Rajagopalan, and M. Stephens, "Effect of 
initial conditions on the near-field development of a round jet," Exp. Fluids 37, 56 (2004).

${ }^{31}$ I. Wygnanski and H. E. Fiedler, "Some measurements in the selfpreserving jet," J. Fluid Mech. 38, 577 (1969).

${ }^{32}$ N. R. Panchapakesan and J. L. Lumley, "Turbulence measurements in axisymmetric jets of air and helium. Part 1. Air jet," J. Fluid Mech. 246, 197 (1993).

${ }^{33}$ R. A. Antonia, B. R. Satyaprakash, and A. K. M. F. Hussain, "Measurements of dissipation rate and some other characteristics of turbulent plane and circular jets," Phys. Fluids 23, 695 (1980).

${ }^{34}$ W. K. George and H. J. Hussein, "Locally axisymmetric turbulence," J. Fluid Mech. 233, 1 (1991).

${ }^{35}$ F. H. Champagne, "The fine-scale structure of the turbulent velocity field," J. Fluid Mech. 86, 67 (1978).

${ }^{36}$ T. Y. Hou, X.-H. Wu, S. Chen, and Y. Zhou, "Effect of finite computational domain on turbulence scaling law in both physical and spectral spaces," Phys. Rev. E 58, 5841 (1998).

${ }^{37}$ R. A. Antonia and R. J. Smalley, "Anomalous scaling of velocity and temperature structure functions," Phys. Rev. E 63, 025301 (2001).

${ }^{38}$ R. A. Antonia, M. Ould-Rouis, F. Anselmet, and Y. Zhu, "Analogy between predictions of Kolmogorov and Yaglom," J. Fluid Mech. 332, 395 (1997).

${ }^{39}$ A. M. Obukhov, "Structure of temperature field in turbulent flows," Izv. Akad. Nauk SSSR, Ser. Geogr. Geofiz. 13, 58 (1949).

${ }^{40} \mathrm{~L}$. Mydlarski and Z. Warhaft, "On the onset of high-Reynolds-number grid-generated wind tunnel turbulence," J. Fluid Mech. 320, 331 (1996).

${ }^{41}$ S. Gamard and W. K. George, "Reynolds number dependence of energy spectra in the overlap region of isotropic turbulence," Flow, Turbul. Combust. 63, 443 (1999).

${ }^{42}$ B. R. Pearson and R. A. Antonia, "Reynolds-number dependence of turbulent velocity and pressure increments," J. Fluid Mech. 444, 343 (2001).

${ }^{43} \mathrm{M}$. Coantic and J.-J. Lasserre, "On pre-dissipative 'bumps' and a Reynolds-number-dependent spectral parameterization of turbulence," Eur. J. Mech. B/Fluids 18, 1027 (1999).

${ }^{44} \mathrm{G}$. Comte-Bellot and S. Corrsin, "Simple Eulerian time correlation of fulland narrow-band velocity signals in grid-generated, 'isotropic' turbulence," J. Fluid Mech. 48, 273 (1971).

${ }^{45} \mathrm{~J}$. Tso and F. Hussain, "Organized motions in a fully developed turbulent axisymmetric jet," J. Fluid Mech. 203, 425 (1989).

${ }^{46}$ H. E. Fiedler, "Coherent structures," in Proceedings of the First European Turbulence Conference, edited by G. Comte-Bellot and J. Mathieu (Springer-Verlag, Berlin, 1987), pp. 320-336.

${ }^{47}$ P. Burattini and L. Djenidi, "Velocity and passive scalar characteristics in a round jet with grids at the nozzle exit," Flow, Turbul. Combust. 72, 199 (2004).

${ }^{48}$ G. P. Romano and R. A. Antonia, "Longitudinal and transverse structure functions in a turbulent round jet: Effect of initial conditions and Reynolds number," J. Fluid Mech. 436, 231 (2001).

${ }^{49} Q(x) f(\xi) \equiv M(x) e(\xi)+2 R(x) h(\xi)$. 